\title{
Shape model, reference system definition, and cartographic mapping standards for comet 67P/Churyumov-Gerasimenko - Stereo-photogrammetric analysis of Rosetta/OSIRIS image data ${ }^{\star}$
}

\author{
F. Preusker ${ }^{1}$, F. Scholten ${ }^{1}$, K.-D. Matz ${ }^{1}$, T. Roatsch ${ }^{1}$, K. Willner ${ }^{1}$, S. F. Hviid ${ }^{1}$, J. Knollenberg ${ }^{1}$, L. Jorda ${ }^{2}$, \\ P. J. Gutiérrez ${ }^{3}$, E. Kührt ${ }^{1}$, S. Mottola ${ }^{1}$, M. F. A'Hearn ${ }^{4}$, N. Thomas ${ }^{5}$, H. Sierks ${ }^{6}$, C. Barbieri ${ }^{7}$, P. Lamy ${ }^{2}$, R. Rodrigo ${ }^{8,9}$, \\ D. Koschny ${ }^{10}$, H. Rickman ${ }^{11,12}$, H. U. Keller ${ }^{13}$, J. Agarwal ${ }^{6}$, M. A. Barucci ${ }^{14}$, J.-L. Bertaux ${ }^{15}$, I. Bertini ${ }^{16}$, \\ G. Cremonese ${ }^{17}$, V. Da Deppo ${ }^{18}$, B. Davidsson ${ }^{11}$, S. Debei ${ }^{19}$, M. De $\mathrm{Cecco}^{20}$, S. Fornasier ${ }^{14,21}$, M. Fulle ${ }^{22}$, \\ O. Groussin ${ }^{2}$, C. Güttler ${ }^{6}$, W.-H. Ip² ${ }^{23}$, J. R. Kramm ${ }^{6}$, M. Küppers ${ }^{24}$, L. M. Lara ${ }^{3}$, M. Lazzarin ${ }^{7}$, J. J. Lopez Moreno ${ }^{3}$, \\ F. Marzari ${ }^{7}$, H. Michalik ${ }^{25}$, G. Naletto ${ }^{26,16,18}$, N. Oklay ${ }^{6}$, C. Tubiana ${ }^{6}$, and J.-B. Vincent ${ }^{6}$
}

(Affiliations can be found after the references)

Received 17 April 2015 / Accepted 2 July 2015

\begin{abstract}
We analyzed more than 200 OSIRIS NAC images with a pixel scale of 0.9-2.4 m/pixel of comet 67P/Churyumov-Gerasimenko (67P) that have been acquired from onboard the Rosetta spacecraft in August and September 2014 using stereo-photogrammetric methods (SPG). We derived improved spacecraft position and pointing data for the OSIRIS images and a high-resolution shape model that consists of about 16 million facets ( $2 \mathrm{~m}$ horizontal sampling) and a typical vertical accuracy at the decimeter scale. From this model, we derive a volume for the northern hemisphere of $9.35 \mathrm{~km}^{3} \pm 0.1 \mathrm{~km}^{3}$. With the assumption of a homogeneous density distribution and taking into account the current uncertainty of the position of the comet's center-of-mass, we extrapolated this value to an overall volume of $18.7 \mathrm{~km}^{3} \pm 1.2 \mathrm{~km}^{3}$, and, with a current best estimate of $1.0 \times 10^{13} \mathrm{~kg}$ for the mass, we derive a bulk density of $535 \mathrm{~kg} / \mathrm{m}^{3} \pm 35 \mathrm{~kg} / \mathrm{m}^{3}$. Furthermore, we used SPG methods to analyze the rotational elements of 67P. The rotational period for August and September 2014 was determined to be $12.4041 \pm 0.0004 \mathrm{~h}$. For the orientation of the rotational axis ( $z$-axis of the body-fixed reference frame) we derived a precession model with a half-cone angle of $0.14^{\circ}$, a cone center position at $69.54^{\circ} / 64.11^{\circ}$ (RA/Dec J2000 equatorial coordinates), and a precession period of 10.7 days. For the definition of zero longitude ( $x$-axis orientation), we finally selected the boulder-like Cheops feature on the big lobe of 67P and fixed its spherical coordinates to $142.35^{\circ}$ right-hand-rule eastern longitude and $-0.28^{\circ}$ latitude. This completes the definition of the new Cheops reference frame for 67P. Finally, we defined cartographic mapping standards for common use and combined analyses of scientific results that have been obtained not only within the OSIRIS team, but also within other groups of the Rosetta mission.
\end{abstract}

Key words. comets: general - planets and satellites: surfaces - reference systems

\section{Introduction}

In March 2004, the European Space Agency (ESA) launched its Rosetta spacecraft to investigate comet 67P/ChuryumovGerasimenko (67P). The Rosetta spacecraft is equipped with 11 orbiter instruments and the lander module Philae. After a ten-year journey that included fly-bys at the Earth, Mars, and the asteroids (2867) Steins and (21) Lutetia (Sierks et al. 2011; Preusker et al. 2012), the Rosetta spacecraft reached 67P at about 3.5 astronomical units (AU) from the Sun and was inserted into a comet-characterization-phase trajectory at the beginning of August 2014. This phase consisted of pyramidal legs, on which Rosetta descended from about $150 \mathrm{~km}$ to approximately $60 \mathrm{~km}$ distance to the nucleus. After this, Rosetta entered a mapping phase (with distances to the surface of about $30 \mathrm{~km}$ ) followed by a close observation phase from distances of 10-20 km in October 2014. In this way, Rosetta approached the target body closer than any other mission to a comet has done

* Appendices are available in electronic form at http: //WwW . aanda.org before (e.g., 1P/Halley, 19P/Borrelly, 9P/Tempel, 103P/Hartley, and $81 \mathrm{P} /$ Wild).

Within the first month of the comet-characterization phase, the Optical, Spectroscopic and Infrared Remote Imaging System (OSIRIS) camera system (Keller et al. 2007) acquired several hundred images of the illuminated surface of 67P (mainly on the northern hemisphere) using the Narrow Angle Camera (NAC) and the Wide Angle Camera (WAC; Sierks et al. 2015). This dataset is of great value for the reconstruction of topographic models (shape models) of the comet's surface with stereo-photogrammetric (SPG) methods. Preliminary versions of SPG shape models have previously been used within many scientific analyses of Rosetta data (El-Maarry et al. 2015; Fornasier et al. 2015; Keller et al. 2015a; Kofman et al. 2015; Massironi et al. 2015; Pajola et al. 2015; Pommerol et al. 2015; La Forgia et al. 2015; Sierks et al. 2015; Thomas et al. 2015b,a; Vincent et al. 2015).

We describe the SPG methods in Sect. 2 and give an overview of all relevant input data for our analysis of Rosetta/OSIRIS NAC image data in Sect. 3. We show the different SPG processing results for 67P (rotational parameters, 
improved orientation data, the final SPG shape model, and estimates for the volume and the bulk density) and perform a quality assessment in Sect. 4. In Sect. 5, we define a new reference coordinate frame for 67P based upon the SPG analysis, and finally propose standards for cartographic mapping of the comet's surface in Sect. 6.

\section{Stereo-photogrammetric methods and their requirements}

In this section, we describe our stereo-photogrammetric approach (SPG) and the requirements related to this. Over the past decades (starting in the 1990s), SPG was successfully applied to many other planetary image datasets to retrieve local, regional, and global shape models from planets, moons, and asteroids of our solar system (e.g., Giese et al. 2006; Gwinner et al. 2009, 2010; Preusker et al. 2012; Scholten et al. 2005, 2012). With some adaptations that address the particular conditions of the Rosetta mission and its main target, we have applied SPG methods for the reconstruction of the irregular shape of the nucleus of comet 67P using OSIRIS NAC image data. The adaptations mainly include a modified preparation of the OSIRIS images for image matching (see Sect. 4), as well as a modified procedure for the generation of the final shape model in three-dimensional coordinates (see Sect. 4.3).

\subsection{Illumination/observation requirements for SPG}

To fully exploit the image data by means of stereophotogrammetric methods, specific requirements with respect to illumination and viewing conditions have to be fulfilled. Variable illumination within stereo datasets has a strong influence on the accuracy and robustness of area-based image-matching techniques (Sect. 2.3), while small stereo viewing angles reduce the quality of the finally reconstructed stereo image geometry (Sect. 2.4). To achieve the best possible quality (accuracy) and quantity (coverage) of the desired 3D data products, it is of central importance to consider these requirements during imagesequence planning under the given mission conditions (specific $\mathrm{s} / \mathrm{c}$ pointing, data volume, downlink capacities, etc.). After the final image acquisition, the dataset has to be reanalyzed with respect to these criteria since the finally acquired dataset may be different in terms of quantity of images (images may have been lost) as well as in terms of the really achieved illumination conditions (particularly problematic for the very irregular shape of 67P). For the final selection of stereo images that feed into the SPG processing, this reanalysis may result in slightly modified criteria (Sect. 3.1), which optimize the accuracy and coverage of the final 3D data products under the conditions of the available dataset. The typical requirements for illumination conditions and observation geometry that should be applied during image-sequence planning are summarized in Table 1 and are discussed with respect to their applicability to the OSIRIS dataset in Sect. 3.1.

\subsection{Stereo-photogrammetric bundle block adjustment}

The central component of the SPG approach is the stereophotogrammetric bundle block adjustment. It comprises a leastsquares adjustment of observations, typically image coordinates of homologous points (tie-points) within (multi-)stereo images, to determine the unknown parameters, that is, six camera orientation parameters (three metric parameters for the camera
Table 1. Illumination and observation requirements for imaging planning.

\begin{tabular}{c|c}
\hline \hline Differences in illumination & $0^{\circ}-10^{\circ}$ \\
\hline Stereo angle & $15^{\circ}-45^{\circ}$ \\
\hline Emission angle & $0^{\circ}-55^{\circ}$ \\
\hline Incidence angle & $5^{\circ}-55^{\circ}$ \\
\hline Phase angle & $5^{\circ}-80^{\circ}$ \\
\hline
\end{tabular}

Notes. These are the best-fit parameters for a subsequent stereo analysis by SPG methods.

position and three angular parameters for the camera pointing) and three coordinates for the tie-point coordinates in object space. Hence, the more image coordinates of tie-points are included, the higher the redundancy within the adjustment process. For the stability of the equation system, each tie-point has to be defined in a set of at least three stereo images (Sect. 4.2). The relation between tie-point coordinates and the corresponding surface point is mathematically defined through so-called collinearity equations (Albertz \& Wiggenhagen 2009) via the orientation and camera calibration data (Sect. 3.2). Initial values for the orientation parameters (nominal position and pointing of the spacecraft) are typically taken from SPICE kernels (Acton 1996). In addition to the orientation data, SPICE kernels also provide information about a priori knowledge for the rotational parameters of the target body, spacecraft clock data, the alignment of the camera to the spacecraft, and geometric calibration parameters of the camera. With sufficient redundancy, the leastsquares optimization within the stereo-photogrammetric bundle block adjustment is capable of correcting for stochastic errors within the observations and is finally capable of precisely determining the orientation parameters of all involved images. In addition, it provides accuracy measures for the image coordinates of the tie-points and for the respective $3 \mathrm{D}$ coordinates on the surface of the observed target body. Systematic errors (e.g., within the a priori information about the rotational parameters of the target body) that are introduced into the adjustment can be analyzed and detected with expanded methods (Sect. 2.5).

\subsection{Tie-point matching}

The determination of image coordinates of tie-points for the block adjustment is based upon the measurement of the positions of identical surface features in different stereo images. For the identification and accurate measurement of these image coordinates, an area-based image-matching algorithm was applied. An accuracy of the tie-point image coordinates of up to 0.1 pixel can be obtained by least-squares image-matching of image patches of 11 to 15 pixels in size (Wewel 1996). At this point, the importance of adequate illumination and observation conditions (Sect. 2.1 and Table 1) within a set of stereo images can be demonstrated. If we consider that all images involved in one set of stereo images have been acquired at different times, the images may contain shadow areas (particularly at low solar incidence angles) that are lacking sufficient image texture for precise image-matching. On the one hand, these shadows may cause voids within the tie-point distribution and may finally reduce the overall coverage of the final $3 \mathrm{D}$ data product. On the other hand, the borders of cast shadows may significantly move between the different image acquisition times and may cause 
gross matching failures. Similarly, high emission angles (observations towards the limb) may cause gross matching failures as well. As a result of the oblique view to the surface, same surface features or areas observed in different images appear very different (up to occlusions near topographic edges) within the matched image patches.

\subsection{Block triangulation}

An entire image block may consist of up to hundreds or thousands of stereo images (see Sects. 4 and 4.2). From such a set of images, we select stereo models (subsets of at least three stereo images that fulfill the illumination and observation requirements). For each stereo model, the derived set of tie-point image coordinates are combined with the geometric calibration and adjusted orientation data to compute 3D object (surface) points by means of an overdetermined forward-ray intersection within a least-squares adjustment. As the result of this final step within the block adjustment, we obtain a set of bodyfixed Cartesian object point coordinates as well as information about the achieved relative accuracy for the retrieved 3D surface points (forward-ray intersection accuracy). The number of tie-points used per stereo model depends on the respective processing step. For the block adjustment, a subset is used to limit the number of observations within the least-squares adjustment (typically still thousands or tens of thousands of points in total). Generally, each image should be represented by at least ten tie-points, which should be well distributed over the image. For 67P, we selected at least 120 tie-points per image, which gives a sufficient redundancy for the least-squares optimization. For the subsequent derivation of a 3D shape model, the entire set of tie-points is used (typically derived for each pixel of the used images, in total up to millions or even billions of points). In either case, the typical 3D point accuracies that can be achieved for the entire triangulated block are, with well-calibrated cameras, with significant image texture for image matching, and with sufficient fulfilment of the illumination and observation requirements, in the order of $10-20 \%$ of the average pixel scale.

\subsection{Brute-force optimization for the determination of rotational elements of target body}

Generally, the block adjustment described in Sect. 2.2 corrects for random components of the errors within initial orientation data. However, the block adjustment is performed in the bodyfixed coordinate system, and therefore the rotational elements of 67P cannot be estimated directly. An insufficiently accurate description of the transformation from the inertial coordinate frame J2000 to the body-fixed coordinate frame induces systematic errors that affect the results of the adjustment. The determination of the rotational elements can now be achieved indirectly from investigations of the remaining stress within the adjusted image block by means of brute-force-like variations of the rotational elements and subsequent analysis of the bundle block adjustment results (e.g., the achieved a posteriori accuracies). This approach can be used to improve the knowledge of the rotational period of the target body and of the orientation of the target rotational axis (expressed by its right ascension and declination with respect to the $\mathrm{J} 2000$ frame), with an iterative procedure (see Sect. 4.1).

With the derived final set of improved measures for the rotational elements from the optimization process, an ultimate block adjustment run is performed to retrieve the final set of adjusted orientation data for all images involved.

\section{Input data for processing of OSIRIS NAC image data using the SPG method}

According to the general procedures and their requirements described above, we now give an overview of the relevant data for the actual processing of OSIRIS NAC images toward the creation of final SPG shape model of 67P.

\subsection{OSIRIS NAC image data}

OSIRIS NAC image data are most valuable for the derivation of a high-resolution shape model of 67P since it is the imaging instrument onboard Rosetta that provides the highest geometrical resolution and also has very high radiometric quality.

To fulfil the illumination and observation requirements (Table 1), we focused on an early mission phase, the cometcharacterization phase, immediately after Rosetta's arrival at 67P. During this phase, the illumination conditions were not ideal but much better in terms of solar incidences than in later phases where the beta angle of Rosetta's orbit (angle between orbit plane and the direction to the Sun) was much higher (up to $60^{\circ}$ ) and resulted in images with extended shadow areas. The selected period lasted from 2014-08-19 until 2014-09-03 and covered about 30 rotations of 67P. The Rosetta orbit trajectory during that time consisted of pyramidal legs at distances of $60-90 \mathrm{~km}$ to the surface of 67P. For this period, we planned to acquire 320 OSIRIS NAC images (the OSIRIS SHAP4S image sequence) in nadir-pointing mode using the NAC orange filter F22 with a central wavelength of $649.2 \mathrm{~nm}$ and a bandwidth of $84.5 \mathrm{~nm}$ and a high signal-to-noise ratio (Keller et al. 2007). Image separation was selected to be roughly every $1 \mathrm{~h}$, with some gaps because of spacecraft operation/maintenance phases. One hour separation (about a twelfth of a full rotational period of $67 \mathrm{P}$ ) provides an angular separation (stereo angle) between subsequent images of about $30^{\circ}$, while additional in between views acquired during the entire period of about two weeks provide additional stereo constellations. Unfortunately, a substantial subset of the envisaged images was not acquired in the planned mode. Hence, for SPG processing, a set of 183 OSIRIS NAC images of the SHAP4S image sequence were finally selected with slightly relaxed criteria for the illumination and observation conditions (stereo angle: $10^{\circ}-45^{\circ}$, incidence angle: $0^{\circ}-60^{\circ}$ ). The typical pixel scale for these images is about $1.0 \mathrm{~m} / \mathrm{pixel}$, with minimum/maximum values of 0.9 and $1.6 \mathrm{~m} / \mathrm{pixel}$.

In addition to these SHAP4S images that were scheduled explicitly for the shape reconstruction by SPG, there are other OSIRIS NAC image sequences from the late-approach phase that are also useful for stereo-photogrammetric analysis. Therefore, we extended the entire image block and selected 26 additional OSIRIS NAC images from the SHAP4 sequence (2014-08-07/08, $1.7 \mathrm{~m} / \mathrm{pixel}$ ) and 9 additional OSIRIS NAC images from the PHASE40 sequence (2014-08-05/06, $2.4 \mathrm{~m} / \mathrm{pixel})$. In the following we use the term SHAP4S as an acronym for the subsequent processing of all image data (PHASE40, SHAP4, and SHAP4S). A list of all 218 images that were selected for the shape reconstruction using SPG is given in Table A.1, and Fig. 1 displays examples for selected NAC images of the different phases.

\subsection{Ancillary data}

As indicated in Sect. 2.1, we retrieved the required ancillary information from SPICE kernels to start the SPG analysis. SPICE kernels (CK and SPK) describe the nominal orientation 
Table 2. Initial SPICE kernels used as input for SPG SHAP4S processing.

\begin{tabular}{ll}
\hline \hline Kernel type & Kernel name \\
\hline 67P ephemeris kernel (SPK) & CORB_DV_075_01__ \\
${ }^{a}$ Rosetta orbit/ephemeris kernel (SPK) & RORB_DV_075_01_00130.BSP \\
${ }^{a}$ Rosetta pointing kernel (CK) & RATT_DV_075_01_01_00130.BSP \\
Rosetta frame kernel (FK) & ROS_V24.TF \\
Rosetta spacecraft clock kernel (SCLK) & ROS_150126_STEP.TSC \\
${ }^{b}$ Rosetta instrument kernel (IK) & No IK kernel available for the OSIRIS NAC \\
${ }^{c}$ Planetary constants kernel (PCK) & No formal PCK kernel for 67P available \\
\hline
\end{tabular}

Notes. ${ }^{(a)}$ These are initial data. For final kernels (improved within SPG), see Table 3. ${ }^{(b)}$ OSIRIS NAC focal length and distortion information for each image pixel were taken from distortion images that have been derived from pre-launch laboratory and in-flight star field measurements (Keller et al. 2007; Tubiana et al. 2015). ${ }^{(c)}$ Initial values for the pole orientation $\left(\mathrm{RA}=69.28^{\circ}, \mathrm{Dec}=64.10^{\circ}\right.$ ) were retrieved from earlier SPG analysis using SHAP4 image data only. The rotational period (W1 $=696.53265399902^{\circ} /$ d, i.e., $12.4043 \mathrm{~h} /$ rotation) was taken from results of analyses of light curves from OSIRIS images of the Rosetta early approach phase (Mottola et al. 2014).

or pointing of the spacecraft $(\mathrm{CK})$, the nominal position of the spacecraft (Rosetta SPK), and the ephemeris (position of the center-of-mass) of the observed target body in the J2000 inertial frame (67P SPK). A SPICE frame kernel (FK) is used to consider information about the alignment of the camera to the spacecraft, while a SPICE instrument kernel (IK) is typically used to retrieve geometric calibration parameters of the camera (e.g., focal length, distortion model). A SPICE kernel containing planetary constants (PCK) provides a priori knowledge about the rotational parameters of the target body, that is, the orientation of the rotation axis of the target body in the $\mathbf{J} 2000$ coordinate frame, the rotational period, probable precession, a zero-longitude definition, etc. Finally, a SPICE kernel about spacecraft clock information (SCLK) and a leap-seconds kernel (LSK) are used to handle image times correctly. Table 2 explicitly lists the initial SPICE kernels that were used as input for the SHAP4S processing.

\section{Derivation of the SHAP4S shape model using SPG}

Out of the 218 selected OSIRIS NAC images, we derived a set of different 321 stereo models with at least three stereo images in each stereo model. From all stereo models we retrieved a total of more than 300 million matched tie-points. The derivation of the tie-points within the OSIRIS images required a modification of the pre-rectification procedure that handles distortions from the very irregular regional and global shape of the nucleus properly. A subset of about 20000 tie-points was used for the first two steps, that is, the refinement of the rotational elements by bruteforce-like optimization (Sect. 4.1) and, applying the derived rotational elements, the subsequent calculation of improved orientation data by block adjustment (Sect. 4.2). The derivation of 3D surface points for all 300 million tie-points and the subsequent data reduction towards the shape model (Sect. 4.3) is based upon the improved rotational elements and orientation data and completes the processing. In Sect. 4.4 we assess the achieved quality of the final SPG SHAP4S shape model.

\subsection{Derivation of refined rotational elements by brute-force-like optimization}

The combination of the SPG method with high-resolution OSIRIS NAC stereo images provides a uniquely high potential for the investigation of 67P's rotational parameters at the meter to sub-meter scale. To optimize the rotational elements, we first investigate the pole orientation (RA and Dec) and its stability over time. With the resulting information about the pole orientation, we then investigate the rotational period. For both steps, we use the achieved a posteriori accuracy of an image block as an indicator for the optimum solution of the respective parameter.

\subsubsection{Pole orientation}

The entire set of images during SHAP4S processing covers a time period of four weeks (2014-08-05 to 2014-09-03). To investigate whether the optimum pole solution is stable or varies over time within this period, we selected six different time intervals (sub-blocks) of about $12-13 \mathrm{~h}$ for which a sufficient number of stereo models are available. Each epoch covers a full rotational period. The selected epochs are on August 6, 8, 21, 28, and 29 and September 2. Specifically for this analysis of the pole orientation, we extended the considered overall time period and included another image sequence (the SHAP2 sequence, about $6 \mathrm{~m} /$ pixel) from August 3. We investigated the stress within the respective sub-blocks separately and derived different optimum solutions for RA and Dec within each subblock by checking the a posteriori accuracy for a set of different RA/Dec combinations within a $1^{\circ} \times 1^{\circ}$ vicinity about the initial values of RA $=69.28^{\circ}, \mathrm{Dec}=64.10^{\circ}$ from the initial PCK (Table 2). Because of the subpixel image-matching accuracy and the high redundancy within each sub-block, a good estimate for a $3 \sigma$ accuracy of these solutions is given by the scale of one NAC pixel for the respective epoch. The optimum solutions and the $3 \sigma$ accuracies are marked in blue in Fig. 2. It can be seen that the optimum RA/Dec positions differ significantly. Obviously, a circle with a particular radius and a well-defined center position can be found that precisely represents the distribution of the different positions with their $3 \sigma$ error ellipses (center position and its estimated $3 \sigma$ accuracy marked in black in Fig. 2). This circle can be understood as a precession cone with a halfdiameter of $0.14^{\circ}$. To use a precession model as an explanation for the distribution of the different pole solutions, we checked whether there is a specific precession period that not only explains the different positions on that precession cone, but also fits to the respective epochs. We found one solution for a precession period at 10.7 days that very well explains the displayed scenario within the uncertainties of the input measures (green dots in Fig. 2 display the model positions on the precession cone using the determined precession period). It is obvious that a static definition of the pole orientation, at any position on the cone or 

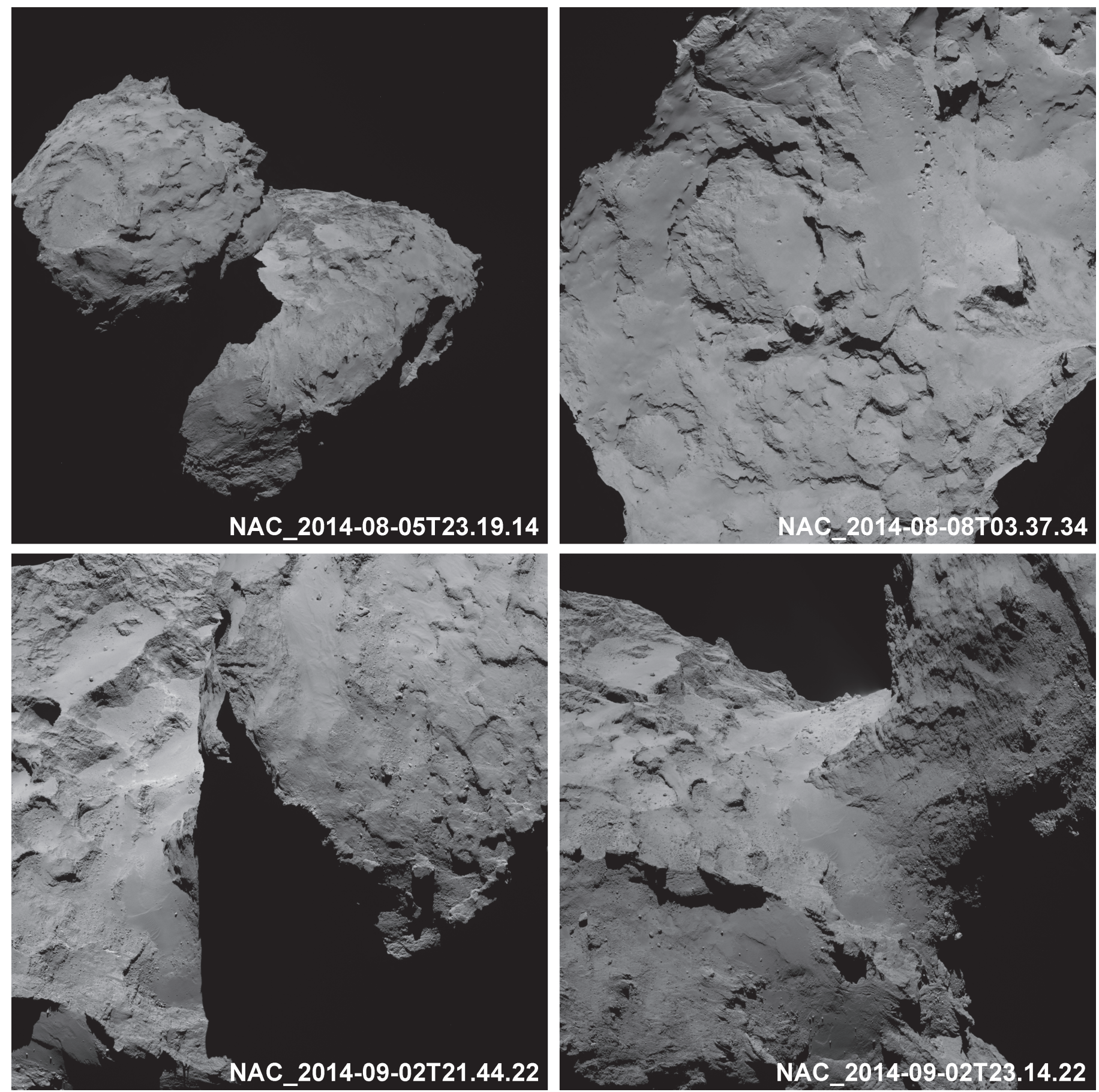

Fig. 1. Examples of OSIRIS NAC images from different epochs that have been used for SPG shape reconstruction. Top left panel: image from PHASE40, top right panel: image from SHAP4, bottom left panel: image from SHAP4S, bottom right panel: image from SHAP4S.

even at its center position, is a less accurate description of the pole orientation than a precession model with the given parameters. With this precession model, the accuracy of the pole orientation over time improves significantly, from about $\pm 5-10 \mathrm{~m}$ up to about $\pm 1 \mathrm{~m}$. Finally, we added four results (orange dots in Fig. 2) from an independent analysis that is based upon landmark tracking (STR). Within their uncertainty, about twice as high as the SPG-based measures, they also fit the precession model. We used this precession model (rounded to significant digits) for the subsequent SPG processing steps. The respective SPICE coding for the derived pole orientation including the precession model into a SPICE planetary constants kernel (PCK) for 67P (Table 3) is the following $(67 \mathrm{P}$ BODYID = 1000012):

BODYID_POLE_RA $=(69.54$ 0. 0.)

BODYID_POLE_DEC $=(64.11$ 0. 0.$)$

BODYID_PM $=\left(\begin{array}{l}114.69696 .543884683 \\ \text { Q. }\end{array}\right)$

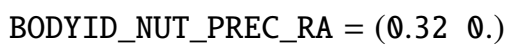

BODYID_NUT_PREC_DEC $=(0.14$ 0.)

BODYID_NUT_PREC_PM $=(-0.288$ 0.)

BODYID_NUT_PREC_ANGLES $=(372.52-1228878.50$ 0. ๑. $)$ 


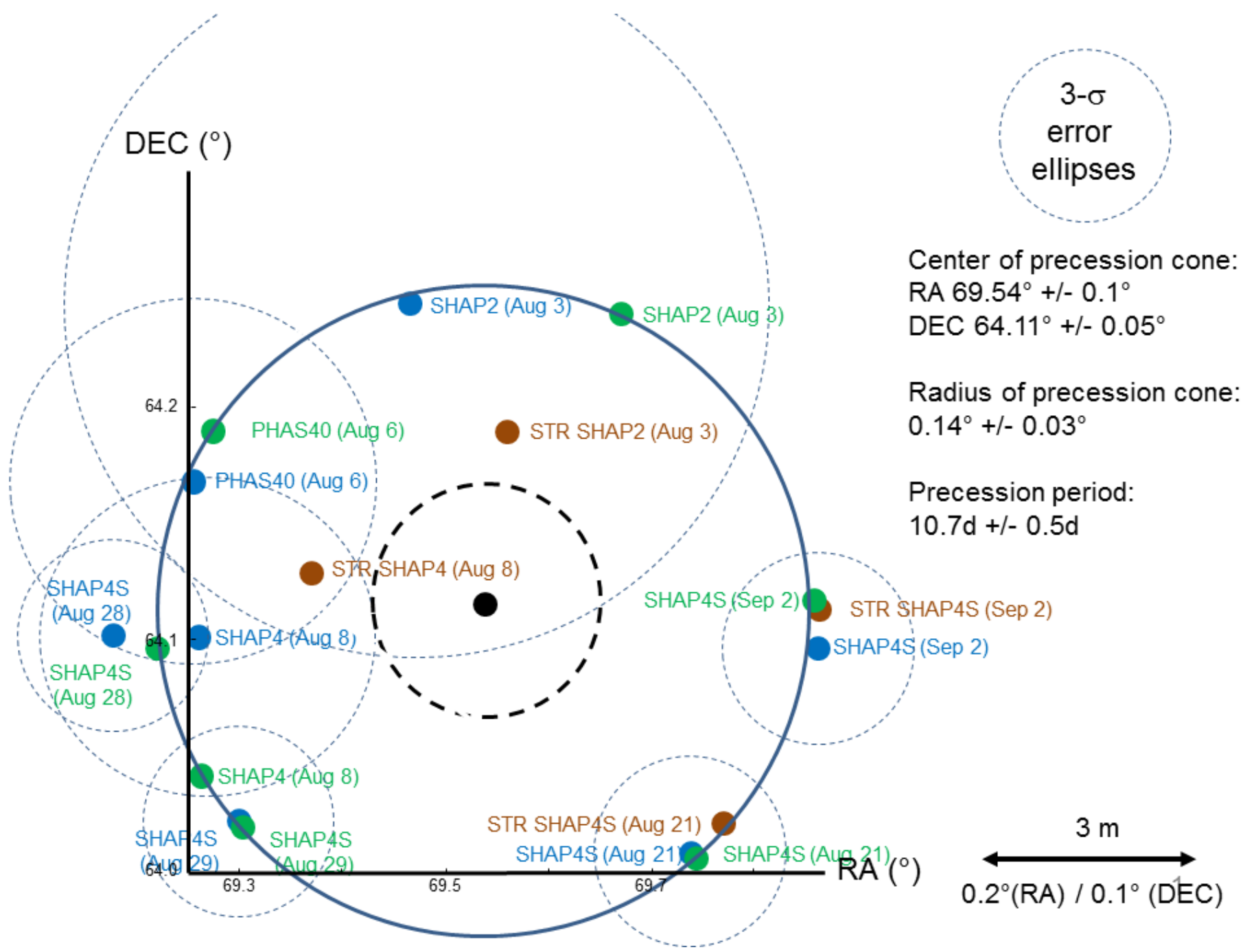

Fig. 2. Analysis of the pole orientation of 67P. Different RA/Dec measurements (blue dots) and respective error ellipses for individual epochs within an overall time period of one month indicate a precession. Nominal RA/Dec values (green dots) for a precession period of 10.7 days are within the error estimates. The black dot and the dashed black circle show the center of the precession cone and its estimated uncertainty.

\subsubsection{Rotational period}

Based on the previously derived rotational model, we applied the same brute-force optimization to the entire SHAP4S block to verify the rotational period. We varied the initial value, $12.4043 \pm 0.0007 \mathrm{~h}$ (Mottola et al. 2014), within a range of $\pm 0.002 \mathrm{~h}$ and again analyzed the a posteriori accuracy of the image block. We found a best-fit rotational period of $12.4041 \pm$ 0.0004 h (i.e., $\mathrm{W} 1=696.543884683^{\circ} /$ day). Within the uncertainty of this analysis, we found no evidence for variations of the rotational period during the investigated time period until early September 2014.

\subsection{Derivation of final improved orientation data by bundle block adjustment}

After eliminating systematic influences from erroneous rotational parameters, it was possible to adjust the stochastic components of errors within the initial orientation data and to determine adjusted orientation data by means of a concluding bundle block adjustment. We used the entire block of 218 OSIRIS NAC images, 321 stereo models, and
20000 tie-points. The forward-ray intersection error of the resulting $3 \mathrm{D}$ surface point coordinates for the tie-points were improved significantly from up to $10-20 \mathrm{~m}$ to finally $\pm 0.3 \mathrm{~m}$ $(1 \sigma)$ on average. This demonstrates that subpixel accuracy has been achieved within the SPG block adjustment, not least because of the integration of the precession model. The mean improvements of the $\mathrm{s} / \mathrm{c}$ position are on the order of $50 \mathrm{~m}$ (up to $120 \mathrm{~m}$ at maximum). The typical amount of the pointing corrections is $0.005^{\circ}-0.01^{\circ}$ for $\mathrm{s} / \mathrm{c}$ pitch and roll (up to $0.025^{\circ}$ at maximum). This corresponds to 5-10 NAC pixels (up to 25 pixels at maximum). For the $\mathrm{s} / \mathrm{c}$ yaw (rotation about the line of sight), the mean correction is about $0.05^{\circ}$ (up to $0.3^{\circ}$ at maximum). For OSIRIS NAC, this corresponds to 1 pixel at the image edges (up to 6 pixels at maximum). The mean values of the corrections for the three pointing angles do not significantly deviate from zero. Thus, there is no indication for an incorrect alignment of the camera to the spacecraft within the SPICE FK frame kernel. Using the previously derived updates of the SPICE planetary constant kernel (PCK), the improved orientation data results were transformed into binary SPICE kernels, a Rosetta pointing kernel (CK), and a Rosetta orbit position kernel (SPK). This set of three new kernels defines 
Table 3. New SPICE kernels derived from SPG SHAP4S processing.

\begin{tabular}{ll}
\hline \hline Kernel type & Kernel name \\
\hline${ }^{a}$ Rosetta orbit kernel (SPK) & cg-dlr_spg-shap4s-v1.0.bsp \\
${ }^{a}$ Rosetta pointing kernel (CK) & cg-dlr_spg-shap4s-v1.0.bc \\
${ }^{b}$ Planetary constants kernel (PCK) & cg-dlr_spg-shap4s-v1.0.tpc \\
\hline
\end{tabular}

Notes. ${ }^{(a)}$ These kernels are not continuous. They provide information only for the discrete epochs of the image acquisition times of images listed in Table A.1. ${ }^{(b)}$ Formally, this kernel is only valid for the epoch 2014-08-05 to 2014-09-03. For application outside this period, we refer to the comments in Sect. 5 .

the first output of the SPG analysis (Table 3). In contrast to the initial kernels, these kernels are not continuous and provide only information for discrete epochs equivalent to the image acquisition times of images listed in Table A.1.

\subsection{Derivation of $3 D$ surface points and data reduction to the final meshed shape model}

Now, with the entire set of geometrical information, which in several aspects is significantly refined by the SPG method, we used the entire set of tie-points and computed body-fixed $x / y / z$ coordinates by forward-ray intersection for more than 300 million $3 \mathrm{D}$ points on the surface of $67 \mathrm{P}$, again with a mean ray intersection accuracy of about $0.3 \mathrm{~m}$. The origin of this bodyfixed coordinate frame is the center-of-mass of $67 \mathrm{P}$ as it is given by the $67 \mathrm{P}$ ephemeris kernel. In contrast to surface reconstruction techniques for more regular bodies (typically done in $2.5 \mathrm{D}$ representations), we had to apply full 3D modeling techniques in the following steps.

To further minimize noise effects, we applied a subsequent filtering that averages $3 \mathrm{D}$ points that fall within voxels of $1 \mathrm{~m}$ in size. Because of the huge number of points that had to be handled, the entire set of points was split into partly overlapping segments of $500 \mathrm{~m}$ in size, and we performed the filtering in each segment. During that filtering, we also considered that the points were derived from images of different mission phases (different pixel scales) and may thus be considered to provide different quality or accuracy. Therefore, we organized the filtered datasets in layers with respect to their pixel scales. Consequently, we only used points from low-resolution image sequences (e.g., PHASE40) where no points from high-resolution sequences or layers (e.g., SHAP4S) were available. After filtering, we merged the segments to one common dataset that consists of about 34 million 3D points at a typical spacing of $1 \mathrm{~m}$.

For the subsequent processing steps toward a meshed shape model, we used the mesh processing system MeshLab ${ }^{\complement}$ (free software $\left.{ }^{1}\right)$. Henceforth, we adopt the commonly used terms "vertex" for a 3D point and "facet" for a list of meshed vertices. Within MeshLab ${ }^{\complement}$, we applied a 2 m clustered vertex subsampling, derived a meshed dataset by Poisson surface reconstruction, and finally removed close vertices (closer than $1 \mathrm{~m}$ ) and retrieved the final meshed SPG SHAP4S shape model. It consists of 8.38 million vertices, 16.75 million facets, and a typical sampling distance of $2 \mathrm{~m}$.

There may be specific applications within scientific disciplines that require reduced versions with significantly fewer vertices or facets for some analyses (e.g., Keller et al. 2015a). We therefore applied the quadratic edge-collapse

\footnotetext{
http://meshlab. sourceforge.net
}

decimation functionality and derived secondary versions of the SPG SHAP4S shape model that are reduced to 4 million facets (about $4 \mathrm{~m}$ average sampling distance), 1 million facets (about $8 \mathrm{~m}$ average sampling distance), 200000 facets (about $16 \mathrm{~m}$ average sampling distance), 100000 facets (about $25 \mathrm{~m}$ average sampling distance), and 50000 facets (about $32 \mathrm{~m}$ average sampling distance). For the reduced versions, we provide average sampling distances since the quadratic decimation function within MeshLab ${ }^{\circledR}$ typically generates lower sampling at smooth areas and retains higher sampling at edges. In Fig. 3 we provide different views of the SPG SHAP4S shape model and of the coordinate axes of the body-fixed reference frame (Sect. 5).

The final dataset, that is, the full-resolution SPG SHAP4S shape model, its reduced versions, the derived SPICE kernels for improved spacecraft position and pointing, and the planetary constants kernel is available online ${ }^{2}$ and will be released via the Planetary Data System (PDS) and the Planetary Science Archive (PSA).

\subsection{Quality assessment}

The quality assessment of a shape model for a previously completely unknown body of a comet is difficult. There is no ground truth available at that resolution that may be used as a reference. Nevertheless, the SPG approach is a completely geometric reconstruction without any assumption about the physical (e.g., photometric) properties of the imaged surface. Therefore, we assume that the derived relative accuracy (at the pixel scale or better) is also a good estimate for the general absolute accuracy. This has been proven within several SPG applications to other stereo image datasets of previous and ongoing planetary missions (e.g., Scholten et al. 2012; Raymond et al. 2012). Thus, SPG results provide very reliable information at the global, regional, and local scale for precise follow-up analyses, for example, about geometrical and gravitational slopes (Groussin et al. 2015). But for the very small scale aspect, we know from experience from other missions (e.g., Preusker et al. 2011) that the effective lateral resolution of the derived 2.5D (Digital Terrain Models, DTM) and 3D (shape models) data products is to some extent limited because of the size of the used image patches within the area-based image-matching process. As a consequence, there is some kind of smoothing effect that limits the effective lateral resolution to typically about 5-10 times the respective image pixel size. This means for the case of the SPG SHAP4S shape model of 67P, as can also be seen within the model, that it provides an effective lateral resolution of about 5-10 m. Hence, topographic features at this size or smaller are not at all or not well represented. Such features are typically small boulders, small depressions (craters), or very local peaklike topographic highs. Of course, the quality of the model also decreases in regions for which no 3D data (points) at all could be derived. These are typically areas that are in permanent shadow in all of the used images. For our 67P data that were acquired in August 2014, these are the extended parts on the southern hemisphere and a few limited areas on the northern hemisphere. Within the 3D shape model, these areas consist of less dense meshes.

With respect to the typical vertical accuracy of the shape model at the lateral scale of $>10 \mathrm{~m}$, we can perform an error propagation from the single point accuracy of $0.3 \mathrm{~m}$ for the 300 million original 3D points to the reduced set of 8.38 million vertices of the meshed shape model. The error propagation yields a noise reduction for the vertical component by a factor

2 http://europlanet.dlr.de/Rosetta 

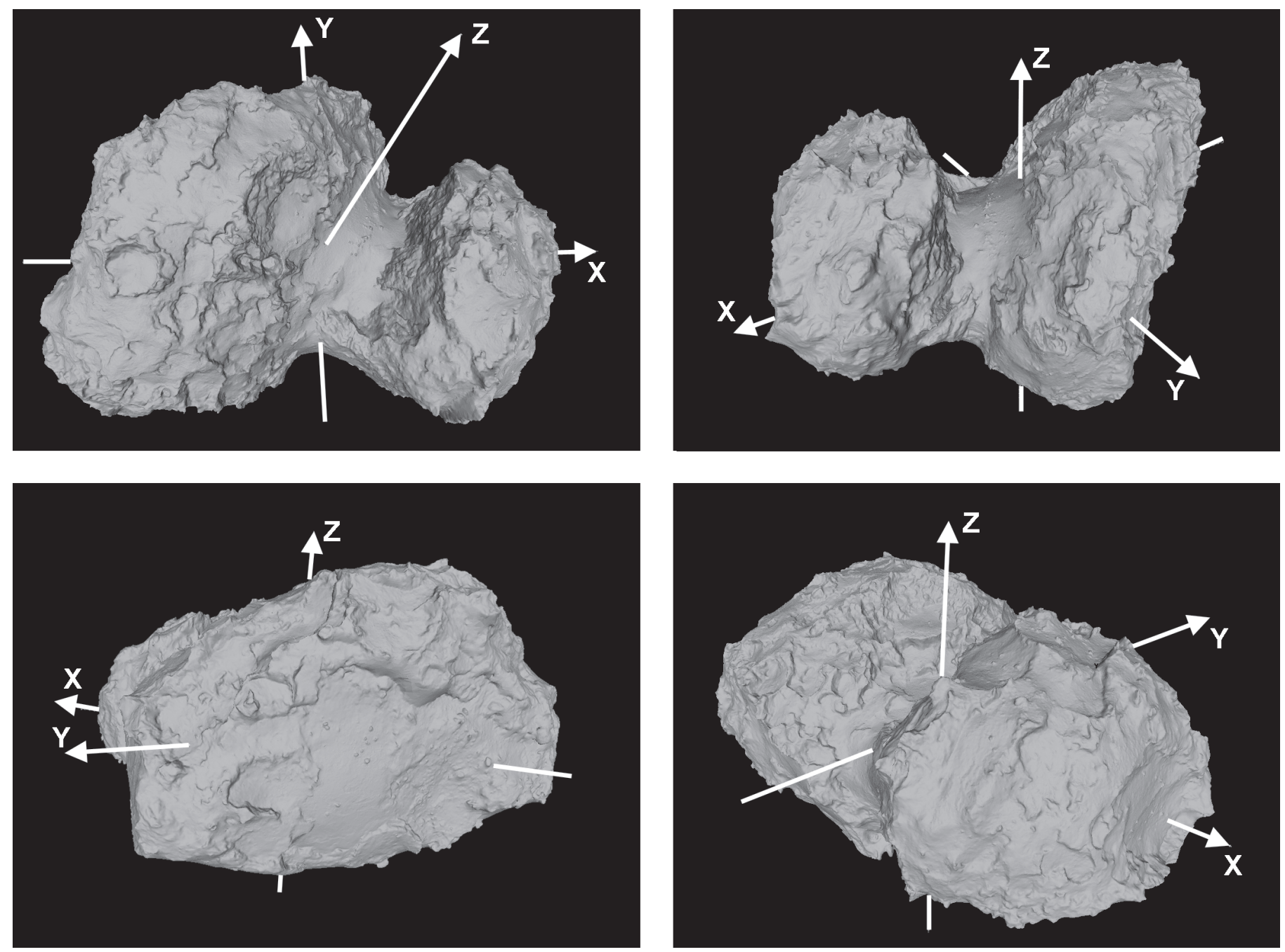

Fig. 3. Different views of the final SPG SHAP4S shape model of 67P and the body-fixed coordinate axes (as described in Sect. 5). Lacking sufficient illumination, major parts of the southern hemisphere are not covered by SPG measurements. The respective areas within the shape model have been interpolated.

of about 6. Therefore, it can be stated that a reliable measure for the vertical accuracy (noise level) of the vertices within the final shape model is at the decimeter scale. The resulting orientations of the facets within the shape model and also geometrical slopes that are derived from this model are typically accurate to about $2-5^{\circ}$.

The value of such a shape model and the importance of the content and of its limitations is dependent on the specific application. Figure 4 provides an impression of the high accuracy at the global, regional, and local scale and also displays the minor limitations at the very local scale. Exemplarily, one of the images used during SPG processing is shown together with a set of additional layers. These layers contain solar incidence angles as well as emission and phase angles that we calculated from the combined set of geometrical information (shape model, improved orientation) for each image pixel, and with pixel to subpixel accuracy. Such datasets have already been used for precise photometric and color analyses of OSIRIS NAC image data (La Forgia et al. 2015; Fornasier et al. 2015).

\subsection{Volume and bulk density}

Major parts of the southern hemisphere of 67P were not illuminated during the August and September 2014 imaging season, and the SPG SHAP4S shape model therefore does not provide accurate shape information for this area. Nevertheless, we can estimate the total volume of $67 \mathrm{P}$ with some assumptions.

The origin of the body-fixed coordinate frame of the shape model is defined by the current estimate for the position of the center-of-mass of 67P. Therefore, we cut the shape model at the equatorial plane and derived a volume for the northern hemisphere of $67 \mathrm{P}$ of $9.35 \mathrm{~km}^{3} \pm 0.1 \mathrm{~km}^{3}(3 \sigma)$. If we conservatively consider the current knowledge of the position of centerof-mass to be uncertain to about $20-50 \mathrm{~m}$ in the $z$-direction, then the measure for the volume of the northern hemisphere increases or decreases by $0.2-0.5 \mathrm{~km}^{3}$ for shifts in the $-z$ or $+z-$ direction. We finally derive a volume of $9.35 \mathrm{~km}^{3} \pm 0.6 \mathrm{~km}^{3}$ $(3 \sigma)$ for the northern hemisphere. Under the assumption that 67P owns a homogeneous density distribution (lacking more detailed knowledge about the interior of $67 \mathrm{P}$ at the time of writing), we can use twice the volume of the northern hemisphere, $18.7 \mathrm{~km}^{3} \pm 1.2 \mathrm{~km}^{3}(3 \sigma)$, as a measure for the overall volume of $67 \mathrm{P}$. With a current estimate of $1.0 \times 10^{13} \mathrm{~kg}$ for the total mass (ESA 2014a), we then derive a bulk density of $535 \mathrm{~kg} / \mathrm{m}^{3} \pm 35 \mathrm{~kg} / \mathrm{m}^{3}(3 \sigma)$, about $15 \%$ higher than previous estimates, $470 \mathrm{~kg} / \mathrm{m}^{3} \pm 45 \mathrm{~kg} / \mathrm{m}^{3}$ (Sierks et al. 2015), but still indicating a high porosity compared to a solid mixture of ice and 

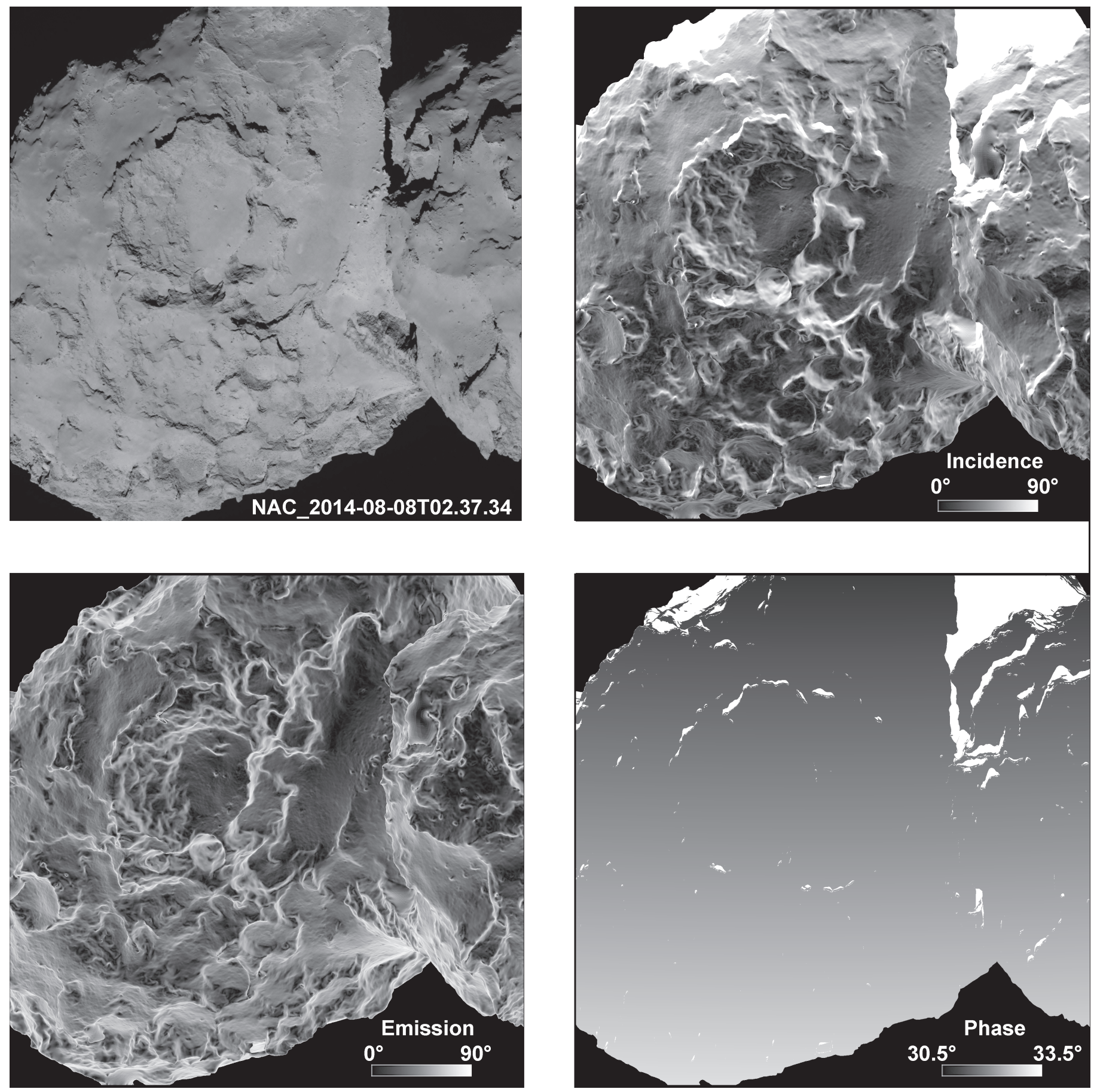

Fig. 4. Solar incidence, emission, and phase angle layers for NAC image (NAC_2014-08-08T02.37.34.580Z_ID20_1397549700_F22). With improved orientation data from SPG and with a high-resolution shape model, this information about the observation and illumination geometry can be derived accurately (to the pixel level) for all images that were included within SPG block adjustment.

non-ice materials. The definition of a best-fit ellipsoid for the particular shape of 67P is questionable. Nevertheless, for completion of the SPICE PCK kernel, we need three radii to define the approximate dimensions of 67P. Because of the unknown shape of the southern hemisphere, the third axis is currently the most undeterminable component of a three-dimensional best-fit ellipsoid. Therefore, we first derived the best-fit values for the equatorial axis and determined the third axis with the criterion that the volume of the best-fit ellipsoid compares to the previously derived volume of $18.7 \mathrm{~km}^{3}$. From this procedure, we retrieved the following SPICE PCK coding (67P BODYID = 1000012) and state explicitly that these values may be inaccurate to about $10 \%$ :

BODYID_RADII $=\left(\begin{array}{lll}2.40 & 1.55 & 1.20\end{array}\right)$.

\section{Definition of the reference system of 67P}

To allow for compatibility among derived data and scientific results from the Rosetta mission, we propose a set of rotational parameters of $67 \mathrm{P}$ that defines a common reference frame for 
the various applications of OSIRIS image data and for common use among other instruments of the Rosetta mission. The following definition of a reference frame and its rotation parameters is based upon the previously described SPG analysis of OSIRIS NAC image data that have been acquired during August and September 2014. Thus, and particularly because of the unpredictable stability of the rotation of a comet nucleus, the relevance of all definitions is formally limited to this time period. The parametrization of the rotational state after that period will require continuous investigation and may extend the definitions given herein.

The main components of the definition of a body-fixed reference frame for 67P have newly been derived within the SPG analysis of the SHAP4S shape model and are described in Sect. 4.2, that is, the mean orientation of the rotation axis at RA/Dec: $69.54^{\circ} / 64.11^{\circ}$ within the inertial J2000 coordinate frame, a precession of the rotation axis of $67 \mathrm{P}$ over a precession cone with a half-cone angle of $0.14^{\circ}$ and centered at the above RA/Dec values, a precession period of $10.7 \mathrm{~d}$, and a rotational period of $12.4041 \mathrm{~h}$, which agrees well with results of light curve analyses (12.4043 h) of OSIRIS NAC/WAC images (Mottola et al. 2014) and ESA/RMOC results (ESA 2014b) for the respective time period based upon NAVCAM and OSIRIS WAC image analysis (12.40428 h, resp. $12.40443 \mathrm{~h})$.

For the alignment of the long equatorial $(x-)$ axis of $67 \mathrm{P}$ with the greatest extension of the figure of 67P, the zero-longitude definition using the W0 parameter (Archinal et al. 2009) was set to $114.69^{\circ}$. This $\mathrm{W} 0$ definition also ensures conformity with the ESA/RMOC reference system where longitudes are implicitly defined by Rosetta 67P ephemeris kernels and body-fixed landmark coordinates. This definition of W0 completes the set of parameters that define the proposed 67P reference frame. The respective coding for the complete definition within a SPICE PCK kernel (Table 3) reads (67P BODYID = 1000012)

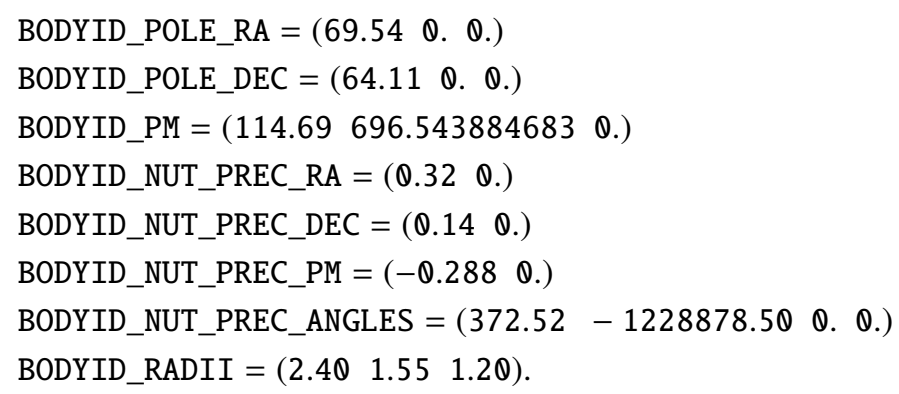

Following these definitions, the center of the prominent boulderlike feature Cheops in the Imhotep region of 67P (Fig. 5, about $50 \mathrm{~m}$ in diameter) on the big lobe of $67 \mathrm{P}$ has the following spherical coordinates (right-hand-rule eastern longitude/centric latitude/radial distance): $142.35^{\circ} /-0.28^{\circ} / 1395 \mathrm{~m}$ and is used as a reference landmark with these fixed coordinates, assuming that its shape and position on the surface of $67 \mathrm{P}$ is stable over the entire duration of the Rosetta mission. The here defined reference system for 67P is called "Cheops reference frame", which is for latitudes, as for longitudes, well within the $0.1^{\circ}$ accuracy level in coincidence with the ESA/RMOC reference frame and body-fixed landmark coordinates. For continuous compatibility of any derived spherical or Cartesian body-fixed coordinates of the surface features of 67P, additional changes of the PM definition of $67 \mathrm{P}$ (e.g., due to any variations of the spin rate (W1) during the mission, as predicted in Keller et al. 2015b) must ensure that the longitude and latitude of Cheops remain fixed (e.g., by a respective adaptation of the W0 value), at least to the current uncertainty of a few meters.
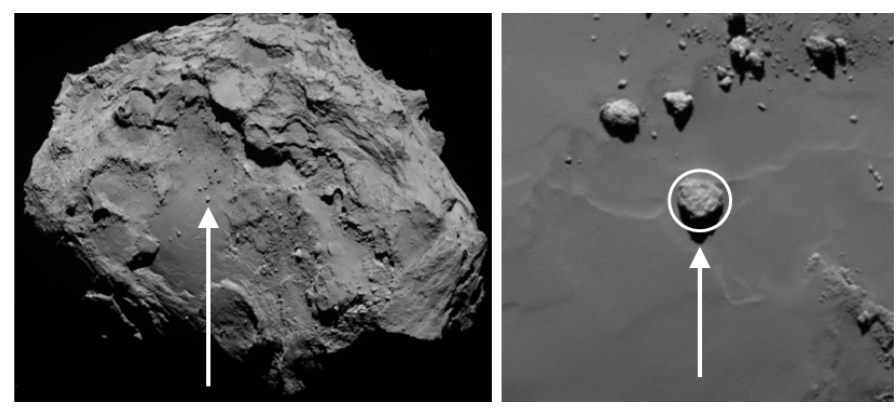

Fig. 5. Boulder-like feature Cheops in the Imhotep region. The center of this feature is the primary feature for the zero-longitude definition of the Cheops reference frame. The context image (left) is a subset of NAC_2014-08-03T16.19.34.554Z_ID20_1397549400_F82, the close-up (right) is taken from NAC_2014-0825T23.12.54.550Z_ID20_1397549700_F22.
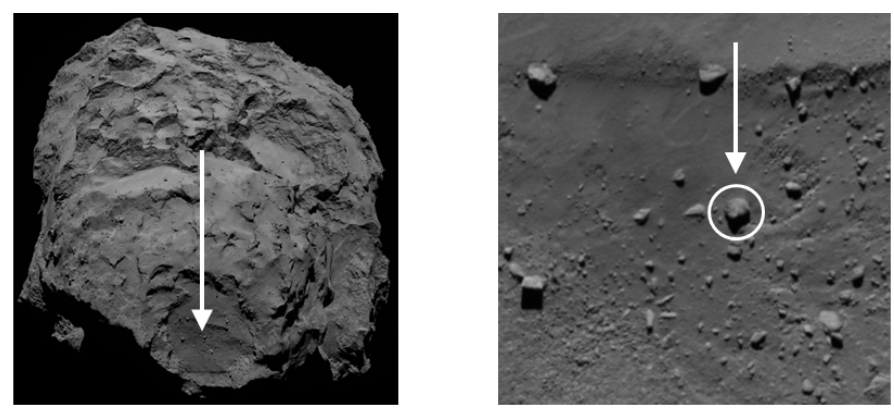

Fig. 6. Boulder-like feature in the Hatmehit region. The center of this feature is the first of two additional secondary features for the zero-longitude definition of the Cheops reference frame. Context image (left): subset of NAC_2014-0806T01.19.14.554Z_ID20_1397549200_F22. Close-up (right): subset of NAC_2014-08-26T04.42.54.592Z_ID20_1397549500_F22.
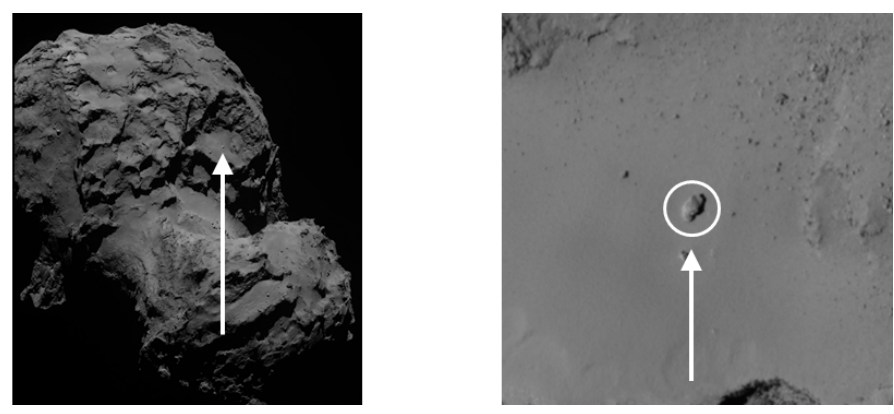

Fig. 7. Boulder-like feature in the Seth region. The center of this feature is the second of two additional secondary features for the zero-longitude definition of the Cheops reference frame. Context image (left): subset of NAC_2014-0806T03.19.48.123Z_ID20_1397549900_F22. Close-up (right): subset of NAC_2014-08-28T18.42.56.579Z_ID20_1397549500_F22.

To further constrain the definition of the Cheops frame, we fixed the center of a secondary boulder-like feature near the equator on the opposite (small) lobe in the Hatmehit region (Fig. 6) at spherical coordinates $354.69^{\circ} / 3.48^{\circ} / 2329 \mathrm{~m}$, and the center of a third high-latitude boulder-like feature in the Seth region (Fig. 7) at $157.82^{\circ} / 71.99^{\circ} / 1099 \mathrm{~m}$. Region names are defined in Sierks et al. (2015) and Thomas et al. (2015b).

Differences between ESA/RMOC and Cheops frame coordinates may only arise in case of new center-of-mass definitions within the ESA/RMOC datasets. Such updates of the centerof-mass definition are currently not expected and will be (if at 
Table 4. Cartesian coordinates of the centers of the local frames for the three entities (SL, BL, and NR).

\begin{tabular}{c|l|c|c|c}
\hline \hline Entity & Proposed SPICE frame & \multicolumn{3}{|c}{ Center coordinates } \\
\hline & & XC $[\mathrm{km}]$ & YC $[\mathrm{km}]$ & $\mathrm{ZC}[\mathrm{km}]$ \\
\hline Global & 67P/C-G_FIXED & 0 & 0 & 0 \\
Big lobe & 67P/C-G_BL_FIXED & -0.42 & 0.26 & -0.06 \\
Small lobe & 67P/C-G_SL_FIXED & 1.48 & -0.34 & 0.25 \\
Neck region & 67P/C-G_NR_FIXED & 0.66 & -0.20 & -0.30 \\
\hline
\end{tabular}

Table 5. Indicative parameters of the two separation planes between the neck region (NR) and the two main lobes (BL and SL).

\begin{tabular}{c|c|c|c}
\hline \hline \multicolumn{4}{c}{ Separation plane BL-NR: $d=a * X+b * Y+c * Z$} \\
\hline$a$ & $b$ & $c$ & $d$ \\
\hline 0.78706205 & -0.55472785 & 0.26983386 & 0.29017997 \\
\hline \multicolumn{4}{c}{ Separation plane SL-NR: $d=a * X+b * Y+c * Z$} \\
\hline$a$ & $b$ & $c$ & $d$ \\
\hline-0.91856635 & 0.29122925 & -0.26724800 & -0.99968761 \\
\hline
\end{tabular}

all) of minor scale (only a few meters, typically in z-direction). Our definition of the Cheops frame is based upon the Rosetta ephemeris of SPICE SPK version 130 (see Table 2) and their relation to the center-of-mass definition from the comet ephemeris of the same SPICE SPK version. Particularly for adequate cartographic mapping applications, it is necessary to subdivide the bilobate shape of 67P into separate entities. Otherwise there is no uniqueness in terms of spherical coordinates (latitude/longitude) for many parts of the entire surface. Therefore, we divided the entire global shape (GL) into three entities (Fig. 8): the small lobe (SL), the big lobe (BL), and the neck region (NR). We defined local frames for each of these three entities. Local frames have their three axes parallel to those of the main 67P bodyfixed frame, but their centers are translated with respect to the center of the 67P body-fixed frame. The Cartesian coordinates of the center of the three local frames are defined in Table 4. In Table 5, we propose explicit parameters for the two separation planes BL-NR and SL-NR. They are considered to be indicative and may be slightly adjusted with respect to the needs of specific applications.

\section{Standards for cartographic mapping of 67P}

For cartographic mapping of the very irregular shape of the nucleus of 67P, we propose standard map projections (Snyder 1987) and map reference bodies for the three entities of 67P. The use of reference spheres as map reference bodies is strongly recommended for the two lobes to avoid discrepancies between different implementations of map projections that are based upon nonspherical reference bodies (oblate spheroids or three-axial ellipsoids). For such nonspherical bodies, there are typically no unique definitions or implementations within the various commonly used mapping software suites.

For the two lobes we propose the Lambert azimuthal map projection (authalic) as the standard map projection. For specific applications, the stereographic map projection (conformal) is proposed as a secondary option. For both map projections, we propose six different standard center coordinates to allow for adequate standardized mapping of the six different aspects or sides of each lobe. For mapping of entire lobes, we propose to use the equidistant projection (also known as simple cylindrical projection or plate carrée).

Since the extension of the specific tube-like neck region (NR) along its cylinder axis is short enough to be approximated by a sphere, the previously described map projections are applicable in almost the same way, except that the two aspects of NR that point towards the BL and SL entities, as well as entire NR equidistant representations, are considered as dispensable.

Although there is no uniqueness for latitude/longitude over the entire body of 67P because of the non-starlike topology of $67 \mathrm{P}$, some applications may require global or hemisphere map representations. For these global maps of $67 \mathrm{P}$, we propose to use the equidistant map projection and north/south polar aspects of the Lambert azimuthal and stereographic map projection. For digital maps and for convenience of any rescaling, the following standard m/pixel values are proposed: $0.25 \mathrm{~m} /$ pixel, $0.5 \mathrm{~m} /$ pixel, $1 \mathrm{~m} /$ pixel, $2 \mathrm{~m} /$ pixel, $4 \mathrm{~m} /$ pixel, $8 \mathrm{~m} /$ pixel, and so on. The radii of the map reference spheres were not chosen as the statistical mean for each entity, but in a way that the maximum deviations from each sphere, and thus distortions from topography, are minimized. Nevertheless, occultations at the local scale may still remain in case of small-scale overhangs. The summary for the definitions for the proposed standard map parameters is listed in Table 6. The following nomenclature for the different maps was defined:

\section{P_RB_R_P_LAT_LON}

$\mathrm{RB}=$ Reference body [GL, BL, SL, or NR]

$\mathrm{R}=$ Radius of the body reference sphere in $\mathrm{m}$

[1500, 1100, or 1000]

$\mathrm{P}=$ Projection

[E = equidistant, $\mathrm{L}=$ Lambert azimuthal, $\mathrm{S}=$ stereographic]

LAT $=$ Center latitude of projection in deg [0 or 90]

$\mathrm{LON}=$ Center longitude of projection in deg.

For illustration, examples for each of the proposed standard maps of 67P are presented in Figs. B.1-B.4.

\section{Conclusion and outlook}

We have successfully applied our stereo photogrammetric SPG methods to OSIRIS NAC image data and derived a highresolution shape model of the irregularly shaped nucleus of comet $67 \mathrm{P} /$ Churyumov-Gerasimenko. A preliminary version of this model has already been used within various scientific applications. SPG methods have also improved the knowledge of the spacecraft position and spacecraft pointing during the investigated period in August and September 2014 and have allowed us to refine the rotational parameters including the derivation of a precession model that was used to define a new reference coordinate frame for the comet. Our definition of standards for cartographic mapping and its application will finally allow valuable comparisons of the diverse scientific results that have been and will be derived within the entire Rosetta mission. Image data that were acquired after September 2014 are available for further refinements of the current shape model. For the expansion of its coverage toward medium and high southern latitudes, OSIRIS NAC image data from future remapping campaigns of the ongoing Rosetta mission will be used. The high accuracy of OSIRIS NAC shape models will probably enable quantifying erosion processes on the surface of a comet for the first time in cometary science and comparing it with recent predictions (Keller et al. 2015a) for 67P/Churyumov-Gerasimenko. 
Table 6. Standard map reference body/dimensions and projections for all entities (67P GL, SL, BL, and NR).

\begin{tabular}{|c|c|c|}
\hline Entity & $\begin{array}{c}\text { Map } \\
\text { reference body }\end{array}$ & $\begin{array}{c}\text { Map projection } \\
\text { map reference coordinates (cen_lat/cen_lon in }{ }^{\circ} \text { ) }\end{array}$ \\
\hline 67P global (GL) & Sphere: radius $1.5 \mathrm{~km}$ & $\begin{array}{l}\text { Entire 67P: equidistant projection (cen_lat/cen_lon: } 0 / 90 \text { ) } \\
\text { Submaps: primary/standard: Lambert azimuthal projection } \\
\text { Submaps: secondary: stereographic projection } \\
2 \text { submap aspects: cen_lat/cen_lon: } 90 / 0,-90 / 0\end{array}$ \\
\hline 67P big lobe (BL) & Sphere: radius $1.5 \mathrm{~km}$ & $\begin{array}{c}\text { Entire lobe: equidistant projection (cen_lat/cen_lon: 0/140) } \\
\text { Submaps: primary/standard: Lambert azimuthal projection } \\
\text { Submaps: secondary: stereographic projection } \\
6 \text { submap aspects: cen_lat/cen_lon: } 90 / 0,-90 / 0,0 / 50,0 / 140,0 / 230,0 / 320\end{array}$ \\
\hline 67P small lobe (SL) & Sphere: radius $1.1 \mathrm{~km}$ & $\begin{array}{c}\text { Entire lobe: equidistant projection (cen_lat/cen_lon: 0/0) } \\
\text { Submaps: primary/standard: Lambert azimuthal projection } \\
\text { Submaps: secondary: stereographic projection } \\
6 \text { submap aspects: cen_lat/cen_lon: } 90 / 0,-90 / 0,0 / 0,0 / 90,0 / 180,0 / 270\end{array}$ \\
\hline $67 \mathrm{P}$ neck region $(\mathrm{NR})$ & Sphere: radius $1.0 \mathrm{~km}$ & $\begin{array}{l}\text { Submaps: primary/standard: Lambert azimuthal projection } \\
\text { Submaps: secondary: stereographic projection } \\
4 \text { submap aspects: cen_lat/cen_lon: } 90 / 0,-90 / 0,0 / 60,0 / 240\end{array}$ \\
\hline
\end{tabular}

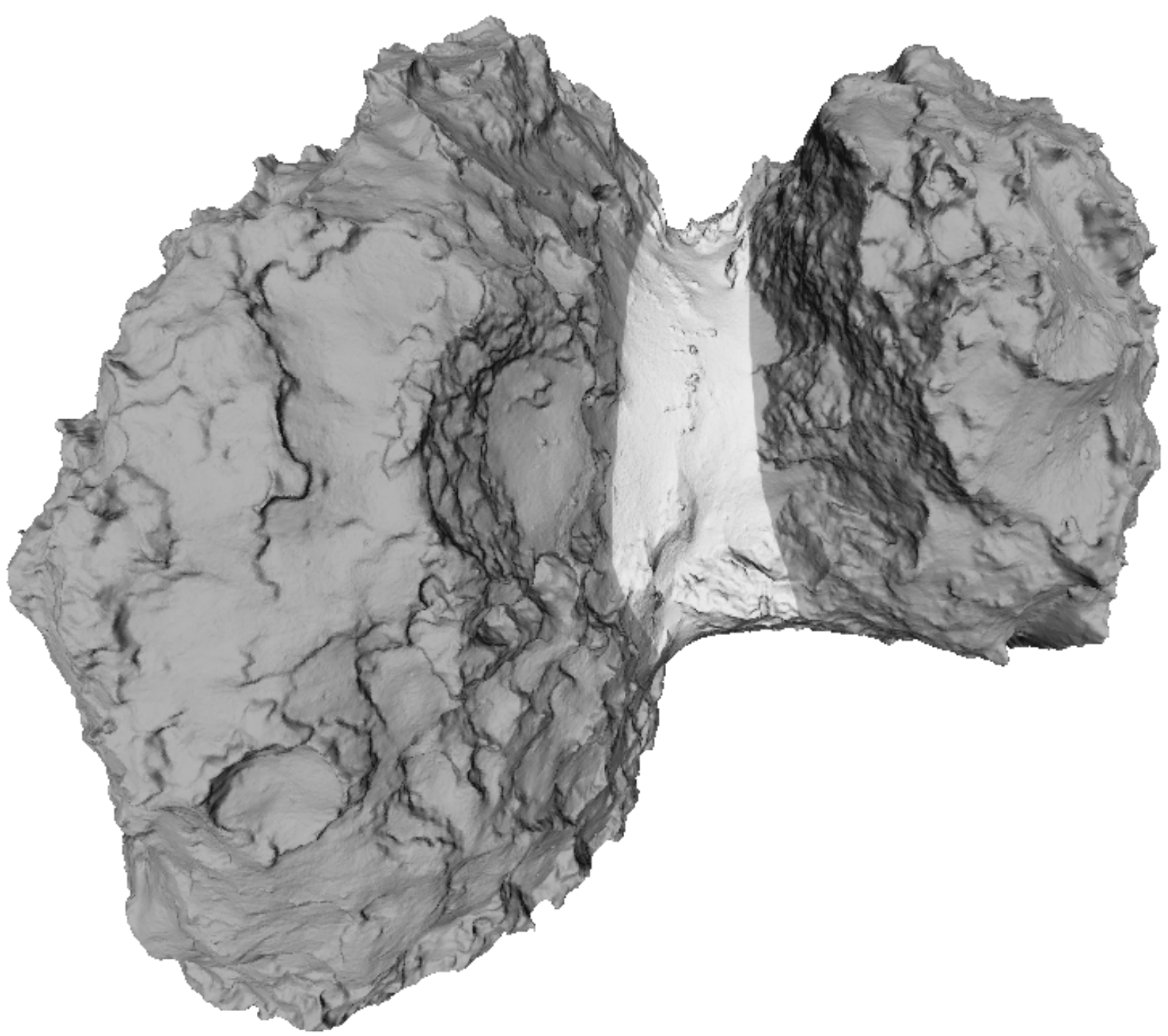

Fig. 8. Views of the three subentities of 67P: Large and small lobe (dark), and neck region (bright). It is obvious that the shapes of these three subentities serve much better as reference bodies for cartographic mapping than the entire body of 67P. 
Acknowledgements. OSIRIS was built by a consortium of the Max-PlanckInstitut für Sonnensystemforschung, Göttingen, Germany, CISAS - University of Padova, Italy, the Laboratoire d'Astrophysique de Marseille, France, the Instituto de Astrofísica de Andalucia, CSIC, Granada, Spain, the Research and Scientific Support Department of the European Space Agency, Noordwijk, The Netherlands, the Instituto Nacional de Técnica Aeroespacial, Madrid, Spain, the Universidad Politéchnica de Madrid, Spain, the Department of Physics and Astronomy of Uppsala University, Sweden, and the Institut für Datentechnik und Kommunikationsnetze der Technischen Universität Braunschweig, Germany. The support of the national funding agencies of Germany (DLR), France (CNES), Italy (ASI), Spain (MEC), Sweden (SNSB), and the ESA Technical Directorate is gratefully acknowledged. We finally thank Nat Bachman (JPL/NAIF) for his very helpful support in the preparation of SPICE planetary constants kernels and the consortium that developed the MeshLab ${ }^{\odot}$ processing system for making it freely available.

\section{References}

Acton, C. H. 1996, Planet. Space Sci., 44, 65

Albertz, J., \& Wiggenhagen, M. 2009, Guide for Photogrammetry and Remote Sensing (Heidelberg, Germany: Wichmann Verlag)

Archinal, B. A., A'Hearn, M. F., Bowell, E., et al. 2009, Celes. Mech. Dyn. Astron., 109, 101

El-Maarry, M. R., Thomas, N., Giacomini, L., et al. 2015, A\&A, 583, A26

ESA 2014a, Determining the mass of comet 67P/C-G, blog entry, ESA, http://blogs.esa.int/rosetta/2014/08/21/

determining-the-mass-of-comet-67pc-g/

ESA 2014b, Issues Rosetta Mission Operations Report, RO-ESC-RP-5018 $183 \& 184$

Fornasier, S., Hasselmann, P. H., Barucci, M. A., et al. 2015, A\&A, 583, A30

Giese, B., Neukum, G., Roatsch, T., Denk, T., \& Porco, C. C. 2006, Planet. Space Sci., 54, 1156

Groussin, O., Jorda, L., Auger, A.-T., et al. 2015, A\&A, 583, A32

Gwinner, K., Scholten, F., Preusker, F., et al. 2010, Earth Planet. Sci. Lett., 294, 506

Gwinner, K., Scholten, F., Spiegel, M., et al. 2009, Photogramm. Eng. Remote Sens., 75, 1127

Keller, H. U., Barbieri, C., Lamy, P., et al. 2007, Space Sci. Rev., 128, 433

Keller, H. U., Mottola, S., Davidsson, B., et al. 2015a, A\&A, 583, A34

Keller, H. U., Mottola, S., Skorov, Y., \& Jorda, L. 2015b, A\&A, 579, L5

Kofman, W., Herique, A., Barbin, Y., et al. 2015, Science, submitted

La Forgia, F., Giacomini, L., Lazzarin, M., et al. 2015, A\&A, 583, A41

Massironi, M., Simioni, E., Marzari, F., et al. 2015, Nature, submitted

Mottola, S., Lowry, S., Snodgrass, C., et al. 2014, A\&A, 569, L2

Pajola, M., Vincent, J.-B., Güttler, C., et al. 2015, A\&A, 583, A37

Pommerol, A., Thomas, N., El-Maarry, M. R., et al. 2015, A\&A, 583, A25

Preusker, F., Oberst, J., Head, J. W., et al. 2011, Planet. Space Sci., 59, 1910

Preusker, F., Scholten, F., Knollenberg, J., et al. 2012, Planet. Space Sci., 66, 54

Raymond, C. A., Jaumann, R., Nathues, A., et al. 2012, The Dawn Mission to

Minor Planets 4 Vesta and 1 Ceres, (New York: Springer Verlag), 163, 487

Scholten, F., Gwinner, K., Roatsch, T., et al. 2005, Photogramm. Eng. Remote Sens., 71, 1143

Scholten, F., Oberst, J., Matz, K.-D., et al. 2012, J. Geophys. Res., 117, e00H17

Sierks, H., Lamy, P., Barbieri, C., et al. 2011, Science, 334, 487

Sierks, H., Barbieri, C., Lamy, P. L., et al. 2015, Science, 347, aaa0440

Snyder, J. P. 1987, Map Projections: A Working Manual, USGS Professional Paper 1395, USGS

Thomas, N., Davidsson, B., El-Maarry, M. R. et al. 2015a, A\&A, 583, A17

Thomas, N., Sierks, H., Barbieri, C., et al. 2015b, Science, 347, aaa0440

Tubiana, C., Güttler, C., Kovacs, G., et al. 2015, A\&A, 583, A46
Vincent, J.-B., Bodewits, D., Besse, S., et al. 2015, Nature, 523, 63 Wewel, F. 1996, Int. Arch. Photogram. Remote Sensing, 31, 936

1 German Aerospace Center (DLR), Institute of Planetary Research, 12489 Berlin, Germany e-mail: Frank.Preusker@dlr.de

2 Aix-Marseille Université, CNRS, LAM (Laboratoire d'Astrophysique de Marseille), UMR 7326, 38 rue Frédéric Joliot-Curie, 13388 Marseille, France

3 Instituto de Astrofisica de Andalucia (CSIC), c/ Glorieta de la Astronomìa s/n, 18008 Granada, Spain

${ }^{4}$ University of Maryland, Department of Astronomy, College Park, MD 20742-2421, USA

5 Physikalisches Institut der Universität Bern, Sidlerstr. 5, 3012 Bern, Switzerland

${ }^{6}$ Max-Planck-Institut für Sonnensystemforschung, Justus-vonLiebig-Weg, 3, 37077, Göttingen, Germany

7 University of Padova, Department of Physics and Astronomy, Vicolo dell'Osservatorio 3, 35122 Padova, Italy

8 Centro de Astrobiología, CSIC-INTA, Torrejón de Ardoz, 28850 Madrid, Spain

9 International Space Science Institute, Hallerstrasse 6, 3012 Bern, Switzerland

10 Research and Scientific Support Department, European Space Agency, 2201 Noordwijk, The Netherlands

11 Department of Physics and Astronomy, Uppsala University, 75120 Uppsala, Sweden

12 PAS Space Reserch Center, Bartycka 18A, 00716 Warszawa, Poland

13 Institut für Geophysik und extraterrestrische Physik (IGEP), Technische Universität 31 Braunschweig, Mendelssohnstr. 3, 38106 Braunschweig, Germany

14 LESIA, Observatoire de Paris, CNRS, UPMC Univ. Paris 06, Univ. Paris-Diderot, 5 place J. Janssen, 92195 Meudon Principal Cedex, France

15 LATMOS, CNRS/UVSQ/IPSL, 11 boulevard d'Alembert, 78280 Guyancourt, France

16 Center of Studies and Activities for Space (CISAS) "G. Colombo", University of Padova, via Venezia 15, 35131 Padova, Italy

17 INAF-Osservatorio Astronomico di Padova, Vicolo dell' Osservatorio 5, 35122 Padova, Italy

18 CNR-IFN UOS Padova LUXOR, via Trasea 7, 35131 Padova, Italy

19 Department of Industrial Engineering - University of Padova, via Venezia 1, 35131 Padova, Italy

${ }^{20}$ UNITN, Universitá di Trento, via Mesiano 77, 38100 Trento, Italy

21 Univ. Paris Diderot, Sorbonne Paris Cité, 4 rue Elsa Morante, 75205 Paris Cedex 13, France

22 INAF-Osservatorio Astronomico di Trieste, via Tiepolo 11, 34143 Trieste, Italy

${ }^{23}$ Institute for Space Science, National Central University, 32054 Chung-Li, Taiwan

${ }^{24}$ ESA/ESAC, PO Box 78, 28691 Villanueva de la Cañada, Spain

25 Institut für Datentechnik und Kommunikationsnetze, Hans-Sommer-Str. 66, 38106 Braunschweig, Germany

26 Department of Information Engineering, University of Padova, via Gradenigo 6/B, 35131 Padova, Italy 
A\&A 583, A33 (2015)

\section{Appendix A: Tables}

Table A.1. List of 218 OSIRIS NAC images used within SPG SHAP4S shape reconstruction.

PDS image names

NAC_2014-08-05T23.19.14.571Z_ID20_1397549800_F22.IMG NAC_2014-08-06T01.19.14.586Z_ID20_1397549200_F22.IMG NAC_2014-08-06T03.19.14.583Z_ID20_1397549600_F22.IMG NAC_2014-08-06T04.19.13.626Z_ID20_1397549300_F22.IMG NAC_2014-08-06T06.19.14.589Z_ID20_1397549700_F22.IMG NAC_2014-08-07T18.37.34.552Z_ID20_1397549700_F22.IMG NAC_2014-08-07T19.37.34.561Z_ID20_1397549500_F22.IMG NAC_2014-08-07T20.37.34.564Z_ID20_1397549300_F22.IMG NAC_2014-08-07T21.37.34.582Z_ID20_1397549100_F22.IMG NAC_2014-08-07T23.37.34.573Z_ID20_1397549300_F22.IMG NAC_2014-08-08T00.37.34.557Z_ID20_1397549100_F22.IMG NAC_2014-08-08T01.37.34.546Z_ID20_1397549900_F22.IMG NAC_2014-08-08T02.37.34.580Z_ID20_1397549700_F22.IMG NAC_2014-08-08T03.37.34.555Z_ID20_1397549500_F22.IMG NAC_2014-08-08T04.37.34.583Z_ID20_1397549300_F22.IMG NAC_2014-08-08T05.37.34.546Z_ID20_1397549100_F22.IMG NAC_2014-08-08T06.37.34.585Z_ID20_1397549900_F22.IMG NAC_2014-08-08T07.37.34.545Z_ID20_1397549700_F22.IMG NAC_2014-08-20T00.42.54.571Z_ID20_1397549100_F22.IMG NAC_2014-08-20T02.42.54.582Z_ID20_1397549500_F22.IMG NAC_2014-08-20T05.42.54.560Z_ID20_1397549100_F22.IMG NAC_2014-08-21T12.42.54.573Z_ID20_1397549000_F22.IMG NAC_2014-08-21T14.42.54.648Z_ID20_1397549400_F22.IMG NAC_2014-08-21T16.42.56.549Z_ID20_1397549300_F22.IMG NAC_2014-08-21T18.42.54.570Z_ID20_1397549700_F22.IMG NAC_2014-08-21T20.42.54.581Z_ID20_1397549100_F22.IMG NAC_2014-08-21T23.42.54.674Z_ID20_1397549700_F22.IMG NAC_2014-08-22T01.41.54.664Z_ID20_1397549000_F22.IMG NAC_2014-08-22T03.42.53.641Z_ID20_1397549000_F22.IMG NAC_2014-08-22T06.42.54.566Z_ID20_1397549600_F22.IMG NAC_2014-08-22T08.42.54.550Z_ID20_1397549000_F22.IMG NAC_2014-08-22T12.42.55.636Z_ID20_1397549500_F22.IMG NAC_2014-08-22T14.42.54.559Z_ID20_1397549900_F22.IMG NAC_2014-08-22T16.42.54.568Z_ID20_1397549300_F22.IMG NAC_2014-08-22T18.42.54.669Z_ID20_1397549700_F22.IMG NAC_2014-08-22T20.42.54.564Z_ID20_1397549100_F22.IMG NAC_2014-08-23T01.42.54.575Z_ID20_1397549800_F22.IMG NAC_2014-08-23T11.42.54.571Z_ID20_1397549500_F22.IMG NAC_2014-08-23T13.42.54.544Z_ID20_1397549900_F22.IMG NAC_2014-08-23T15.42.54.636Z_ID20_1397549300_F22.IMG NAC_2014-08-23T18.42.56.581Z_ID20_1397549400_F22.IMG NAC_2014-08-23T20.42.54.572Z_ID20_1397549800_F22.IMG NAC_2014-08-23T23.42.54.544Z_ID20_1397549600_F22.IMG NAC_2014-08-24T01.42.54.581Z_ID20_1397549000_F22.IMG NAC_2014-08-24T03.42.54.692Z_ID20_1397549000_F22.IMG NAC_2014-08-24T06.42.54.590Z_ID20_1397549500_F22.IMG NAC_2014-08-25T11.12.56.551Z_ID20_1397549800_F22.IMG NAC_2014-08-25T12.42.54.560Z_ID20_1397549000_F22.IMG NAC_2014-08-25T14.42.54.574Z_ID20_1397549600_F22.IMG NAC_2014-08-25T16.42.54.555Z_ID20_1397549200_F22.IMG NAC_2014-08-25T19.12.54.557Z_ID20_1397549000_F22.IMG NAC_2014-08-25T20.42.56.587Z_ID20_1397549700_F22.IMG NAC_2014-08-25T23.12.54.550Z_ID20_1397549700_F22.IMG NAC_2014-08-26T00.42.54.555Z_ID20_1397549900_F22.IMG NAC_2014-08-26T02.42.54.581Z_ID20_1397549700_F22.IMG NAC_2014-08-26T04.42.54.592Z_ID20_1397549500_F22.IMG NAC_2014-08-26T06.42.56.564Z_ID20_1397549200_F22.IMG NAC_2014-08-26T08.42.54.556Z_ID20_1397549000_F22.IMG NAC_2014-08-26T12.42.54.588Z_ID20_1397549100_F22.IMG NAC_2014-08-26T14.42.54.578Z_ID20_1397549500_F22.IMG NAC_2014-08-27T03.42.54.575Z_ID20_1397549500_F22.IMG NAC_2014-08-27T07.08.54.591Z_ID20_1397549300_F22.IMG
NAC_2014-08-06T00.19.14.558Z_ID20_1397549500_F22.IMG NAC_2014-08-06T02.19.14.570Z_ID20_1397549900_F22.IMG NAC_2014-08-06T03.19.14.583Z_ID20_1397549600_F22.IMG NAC_2014-08-06T05.19.14.575Z_ID20_1397549000_F22.IMG NAC_2014-08-07T18.20.34.555Z_ID20_1397549300_F22.IMG NAC_2014-08-07T19.20.34.558Z_ID20_1397549100_F22.IMG NAC_2014-08-07T20.20.34.562Z_ID20_1397549900_F22.IMG NAC_2014-08-07T21.20.34.586Z_ID20_1397549700_F22.IMG NAC_2014-08-07T23.20.34.548Z_ID20_1397549900_F22.IMG NAC_2014-08-08T00.20.34.562Z_ID20_1397549700_F22.IMG NAC_2014-08-08T01.21.35.540Z_ID20_1397549500_F22.IMG NAC_2014-08-08T02.20.34.563Z_ID20_1397549300_F22.IMG NAC_2014-08-08T03.20.34.562Z_ID20_1397549100_F22.IMG NAC_2014-08-08T04.20.34.577Z_ID20_1397549900_F22.IMG NAC_2014-08-08T05.20.34.560Z_ID20_1397549700_F22.IMG NAC_2014-08-08T06.20.34.578Z_ID20_1397549500_F22.IMG NAC_2014-08-08T07.20.34.578Z_ID20_1397549300_F22.IMG NAC_2014-08-19T23.42.56.593Z_ID20_1397549900_F22.IMG NAC_2014-08-20T01.42.54.569Z_ID20_1397549300_F22.IMG NAC_2014-08-20T03.42.54.554Z_ID20_1397549700_F22.IMG NAC_2014-08-21T11.42.53.641Z_ID20_1397549800_F22.IMG NAC_2014-08-21T13.42.54.561Z_ID20_1397549200_F22.IMG NAC_2014-08-21T15.41.54.586Z_ID20_1397549000_F22.IMG NAC_2014-08-21T17.42.54.678Z_ID20_1397549500_F22.IMG NAC_2014-08-21T19.42.54.558Z_ID20_1397549900_F22.IMG NAC_2014-08-21T21.42.54.553Z_ID20_1397549300_F22.IMG NAC_2014-08-22T00.42.54.576Z_ID20_1397549900_F22.IMG NAC_2014-08-22T02.42.56.588Z_ID20_1397549800_F22.IMG NAC_2014-08-22T05.42.54.583Z_ID20_1397549400_F22.IMG NAC_2014-08-22T07.42.54.574Z_ID20_1397549800_F22.IMG NAC_2014-08-22T12.12.54.589Z_ID20_1397549000_F22.IMG NAC_2014-08-22T13.42.54.679Z_ID20_1397549700_F22.IMG NAC_2014-08-22T15.42.54.581Z_ID20_1397549100_F22.IMG NAC_2014-08-22T17.42.54.587Z_ID20_1397549500_F22.IMG NAC_2014-08-22T19.42.54.652Z_ID20_1397549900_F22.IMG NAC_2014-08-22T21.41.54.592Z_ID20_1397549000_F22.IMG NAC_2014-08-23T11.12.53.680Z_ID20_1397549300_F22.IMG NAC_2014-08-23T12.42.54.577Z_ID20_1397549700_F22.IMG NAC_2014-08-23T14.42.54.580Z_ID20_1397549100_F22.IMG NAC_2014-08-23T16.42.54.582Z_ID20_1397549500_F22.IMG NAC_2014-08-23T19.42.53.641Z_ID20_1397549600_F22.IMG NAC_2014-08-23T23.12.54.559Z_ID20_1397549400_F22.IMG NAC_2014-08-24T00.42.54.645Z_ID20_1397549800_F22.IMG NAC_2014-08-24T02.42.54.551Z_ID20_1397549200_F22.IMG NAC_2014-08-24T04.42.55.635Z_ID20_1397549100_F22.IMG NAC_2014-08-24T07.08.54.564Z_ID20_1397549700_F22.IMG NAC_2014-08-25T11.42.54.552Z_ID20_1397549200_F22.IMG NAC_2014-08-25T13.42.54.547Z_ID20_1397549800_F22.IMG NAC_2014-08-25T15.42.54.572Z_ID20_1397549400_F22.IMG NAC_2014-08-25T17.42.54.568Z_ID20_1397549400_F22.IMG NAC_2014-08-25T19.42.54.578Z_ID20_1397549000_F22.IMG NAC_2014-08-25T21.42.54.545Z_ID20_1397549900_F22.IMG NAC_2014-08-25T23.42.54.567Z_ID20_1397549300_F22.IMG NAC_2014-08-26T01.42.54.658Z_ID20_1397549100_F22.IMG NAC_2014-08-26T03.42.54.569Z_ID20_1397549300_F22.IMG NAC_2014-08-26T05.41.54.580Z_ID20_1397549000_F22.IMG NAC_2014-08-26T07.42.54.573Z_ID20_1397549400_F22.IMG NAC_2014-08-26T09.42.54.579Z_ID20_1397549600_F22.IMG NAC_2014-08-26T13.42.54.676Z_ID20_1397549300_F22.IMG NAC_2014-08-26T15.42.54.585Z_ID20_1397549000_F22.IMG NAC_2014-08-27T06.42.54.561Z_ID20_1397549100_F22.IMG NAC_2014-08-27T15.42.54.547Z_ID20_1397549200_F22.IMG 
Table A.1. continued.

\begin{tabular}{|c|c|}
\hline image names & \\
\hline NAC_2014-08-28T07.12.53.547Z_ID20_1397549900_F22.IMG & NAC_2014-08-28T07.42.54.602Z_ID20_1397549000_F22.IMG \\
\hline NAC_2014-08-28T08.42.56.549Z_ID20_1397549800_F22.IMG & NAC_2014-08-28T11.12.53.656Z_ID20_1397549400_F22.IMG \\
\hline NAC_2014-08-28T11.42.54.557Z_ID20_1397549600_F22.IMG & NAC_2014-08-28T12.42.54.563Z_ID20_1397549800_F22.IMG \\
\hline NAC_2014-08-28T13.42.54.579Z_ID20_1397549000_F22.IMG & NAC_2014-08-28T14.42.54.590Z_ID20_1397549200_F22.IMG \\
\hline NAC_2014-08-28T15.42.54.550Z_ID20_1397549400_F22.IMG & NAC_2014-08-28T16.42.54.571Z_ID20_1397549600_F22.IMG \\
\hline NAC_2014-08-28T17.41.54.682Z_ID20_1397549000_F22.IMG & NAC_2014-08-28T18.42.56.579Z_ID20_1397549500_F22.IMG \\
\hline NAC_2014-08-28T19.42.54.556Z_ID20_1397549700_F22.IMG & NAC_2014-08-28T20.42.53.590Z_ID20_1397549900_F22.IMG \\
\hline NAC_2014-08-28T21.42.54.554Z_ID20_1397549100_F22.IMG & NAC_2014-08-28T23.12.54.572Z_ID20_1397549500_F22.IMG \\
\hline _1397549700_F22.IMG & NAC_2014-08-29T01.42.54.551Z_ID20_1397549100_F22.IMG \\
\hline 397549300_F22.IMG & -29T03.42.54.576Z_ID20_1397549000_F22.IMG \\
\hline NAC_2014-08-29T04.42.56.583Z_ID2 & NAC_2014-08-29T05.42.54.570Z_ID20_1397549400_F22.IMG \\
\hline NAC_2014-08-29T07.12.54 & NAC_2014-08-29T07.42.54.589Z_ID20_13 \\
\hline NAC_2014 & NAC_20 \\
\hline NAC_201 & MG \\
\hline NAC_2014 & NAC_201 \\
\hline NAC_201 & NAC_20 \\
\hline NAC_201 & NAC_20 \\
\hline NAC_201 & NAC_20 \\
\hline 201 & NAC_20 \\
\hline MG & 00_F22.IMG \\
\hline _2014-0 & 444Z_ID20_1397549800_F22.IMG \\
\hline _F2 & 200_F22.IMG \\
\hline IG & )_F22.IMG \\
\hline IG & NAC_20 \\
\hline & MG \\
\hline NAC_201 & 00_F22.IMG \\
\hline NAC_20 & NAC_20 \\
\hline NA & MG \\
\hline & MG \\
\hline NA & MG \\
\hline NA & MG \\
\hline NA & _F22.IMG \\
\hline _F22.IMG & NAC_2014-09-02T00.42.52.644Z_ID \\
\hline NAC_2014-09-02T01.4 & NAC_2014-09-02T02.42.53.543Z_ID20_1397549100_F22.IMG \\
\hline _2014-09-02T & NAC_2014-09-02T04.42.53.568Z_ID2 \\
\hline _F22.IMG & $-02 \mathrm{~T} 06.42 .52 .638 \mathrm{Z} \_\mathrm{ID}$ \\
\hline 02Т07 4253 53 & 800_F22.IMG \\
\hline C 2014-09-02T12 10225527 & $12.44 .22 .580 \mathrm{Z}$ ID \\
\hline MG & $548 Z$ ID20 139754 \\
\hline MG & NAC_2014-09-02T16.44.2 \\
\hline MG & NAC_201 \\
\hline NAC_2014-09-02T19.44.2 & NAC_2014-09-02T20.44.22.567Z_ID20_1397549600_F22.IMG \\
\hline NAC_2014-09-02T21.44.22.575Z_ID20_1397549800_F22.IMG & NAC_2014-09-02T23.14.22.584Z_ID20_1397549300_F22.IMG \\
\hline NAC_2014-09-02T23.44.22.550Z_ID20_139 & NAC_2014-09-03T00.44.22.553Z_ID20_13马 \\
\hline NAC_2014-09-03T01.44.22.585Z_ID20_13 & NAC_2014-09-03T02.44.22.548Z_ID20_13C \\
\hline NAC_2014-09-03T03.44.22.640Z_ID20_13 & NAC_2014-09-03T04.44.24.553Z_ID20_1397549000_F22.IMG \\
\hline NAC_2014-09-03T05.44.22.555Z_ID20_1397549200_F22.IMG & NAC_2014-09-03T06.44.22.578Z_ID20_1397549400_F22.IMG \\
\hline
\end{tabular}




\section{Appendix B: Figures}
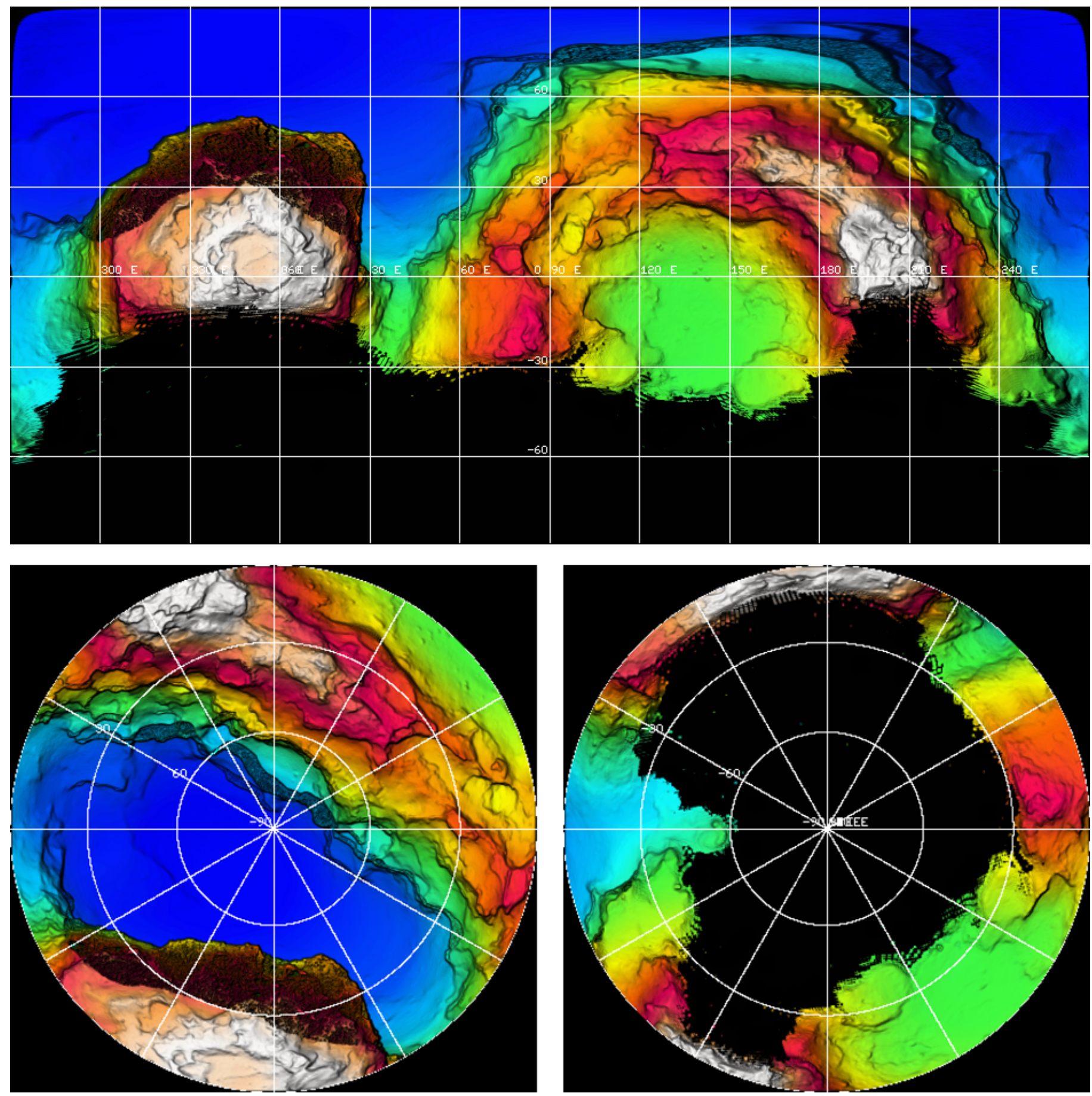

Fig. B.1. Standard maps for GL (entire 67P): if printed in A4 paper format, the map scale is roughly 1:50 K. Height color coding above the $1500 \mathrm{~m}$ reference sphere: blue $=-1000 \mathrm{~m}$ to red/white $=1000 \mathrm{~m}$. Black areas indicate currently not illuminated regions on the southern hemisphere of 67P. Top panel: 67P_GL_1500_E_0_90, bottom left panel: 67P_GL_1500_L_90_0, bottom right panel: 67P_GL_1500_L_-90_0. 
F. Preusker et al.: Shape model of 67P/Churyumov-Gerasimenko
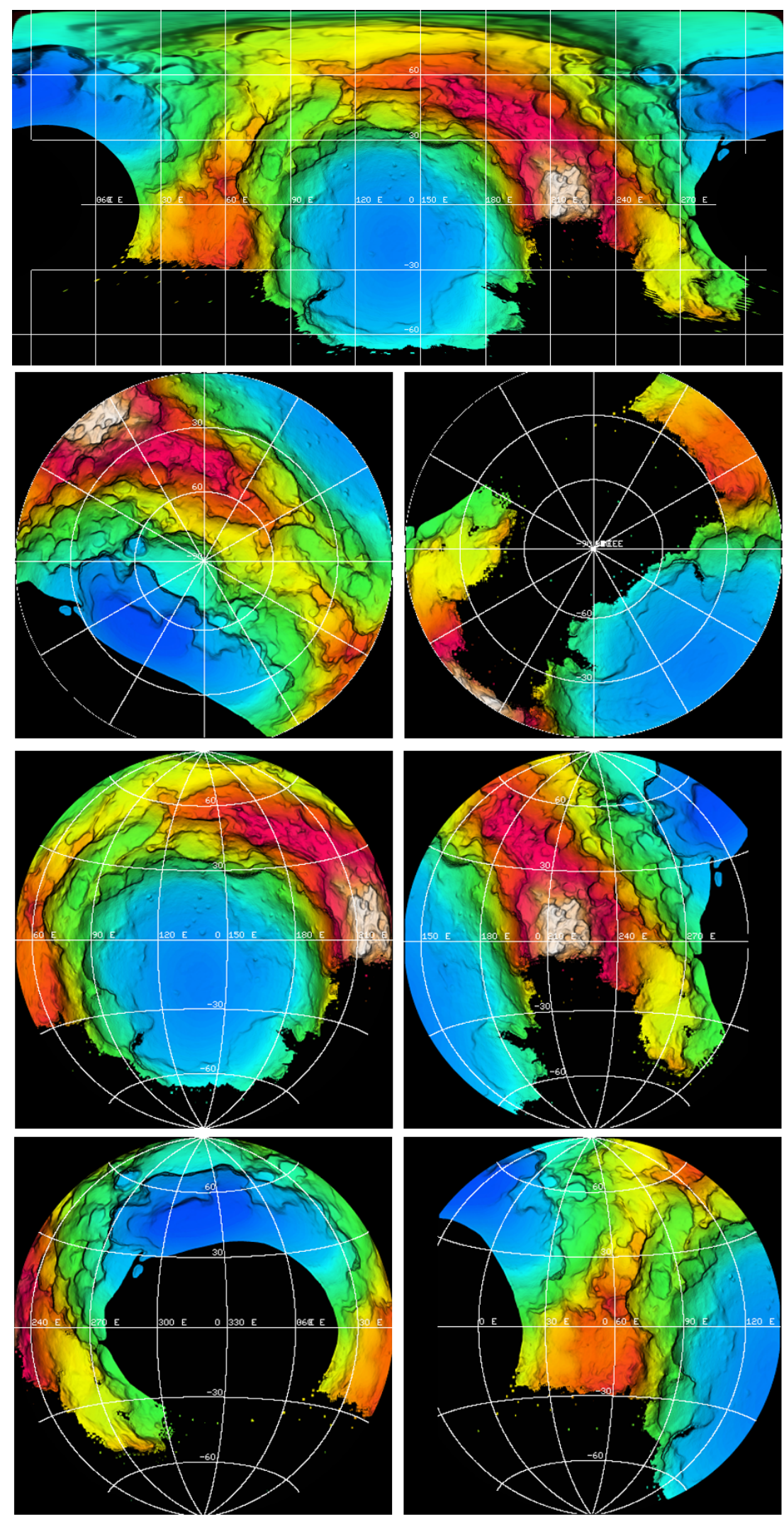

Fig. B.2. Standard maps for the BL (big lobe of 67P): if printed in A4 paper format, the map scale is roughly 1:50 K. Height color coding above the $1500 \mathrm{~m}$ reference sphere: blue $=-1000 \mathrm{~m}$ to red/white $=1000 \mathrm{~m}$. Black areas indicate currently not illuminated unmapped regions on the southern hemisphere of 67P and cut regions to adjacent sublobes (e.g., at the left and right side in 67P_BL_1500_E_0_140). Top panel: 67P_BL_1500_E_0_140, top left panel: 67P_BL_1500_L_90_0, top right panel: 67P_BL_1500_L_-90_0, middle left panel: 67P_BL_1500_L_0_140, middle right panel: 67P_BL_1500_L_0_230, bottom left panel: 67P_BL_1500_L_0_320, bottom right panel: 67P_BL_1500_L_0_50. 

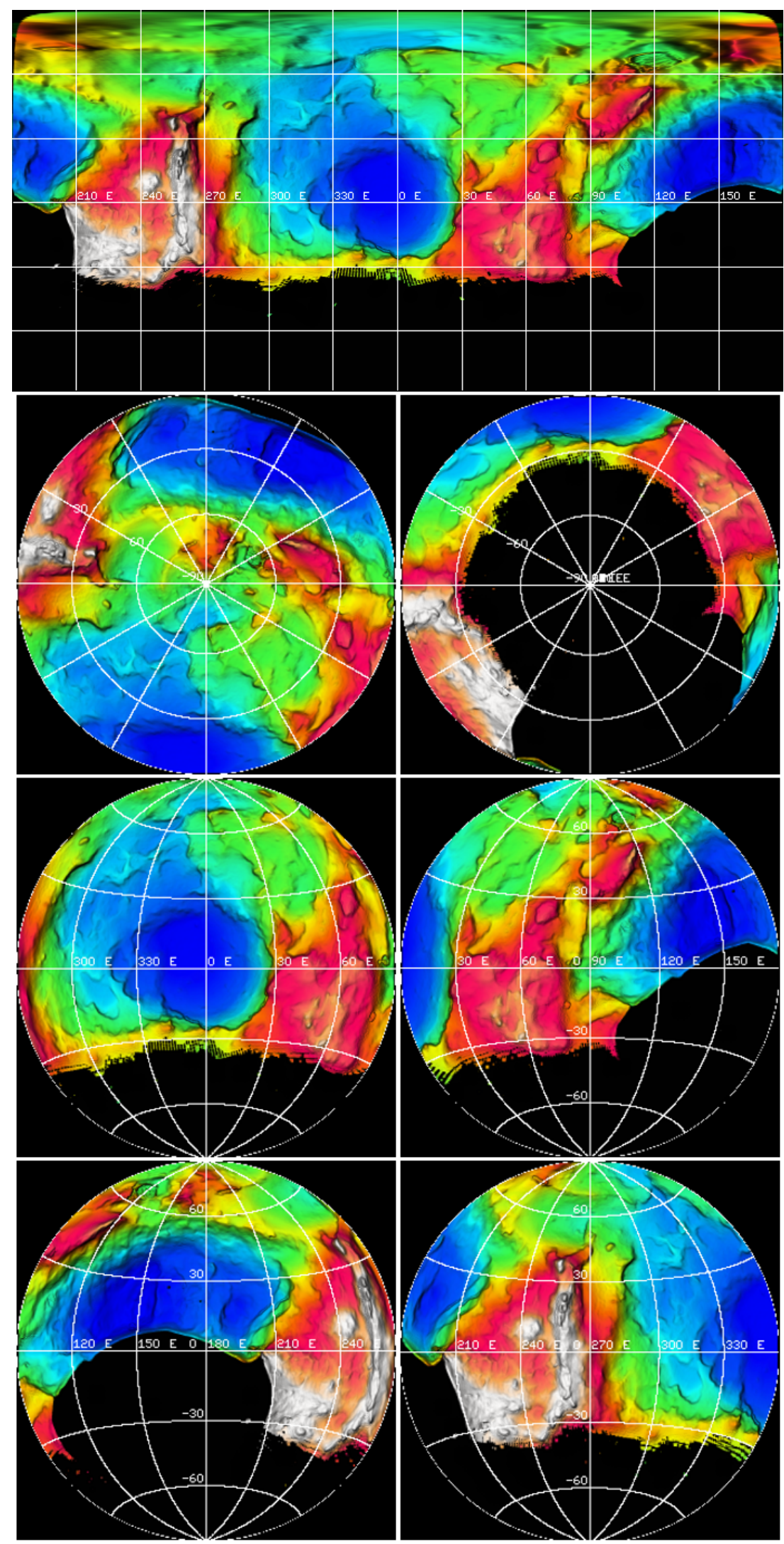

Fig. B.3. Standard maps for the SL (small lobe of 67P): if printed in A4 paper format, the map scale is roughly 1:50 K. Height color coding above the $1100 \mathrm{~m}$ reference sphere: blue $=-300 \mathrm{~m}$ to red/white $=300 \mathrm{~m}$. Black areas indicate currently not illuminated unmapped regions on the southern hemisphere of 67P and cut regions to adjacent sublobes (e.g., at the left and right side in 67P_SL_1100_E_0_0). Top panel: 67P_SL_1100_E_0_0, top left panel: 67P_SL_1100_L_90_0, top right panel: 67P_SL_1100_L_-90_0, middle left panel: 67P_SL_1100_L_0_0, middle right panel: 67P_SL_1100_L_0_90, bottom left panel: 67P_SL_1100_L_0_180, bottom right panel: 67P_SL_1100_L_0_270. 
F. Preusker et al.: Shape model of 67P/Churyumov-Gerasimenko
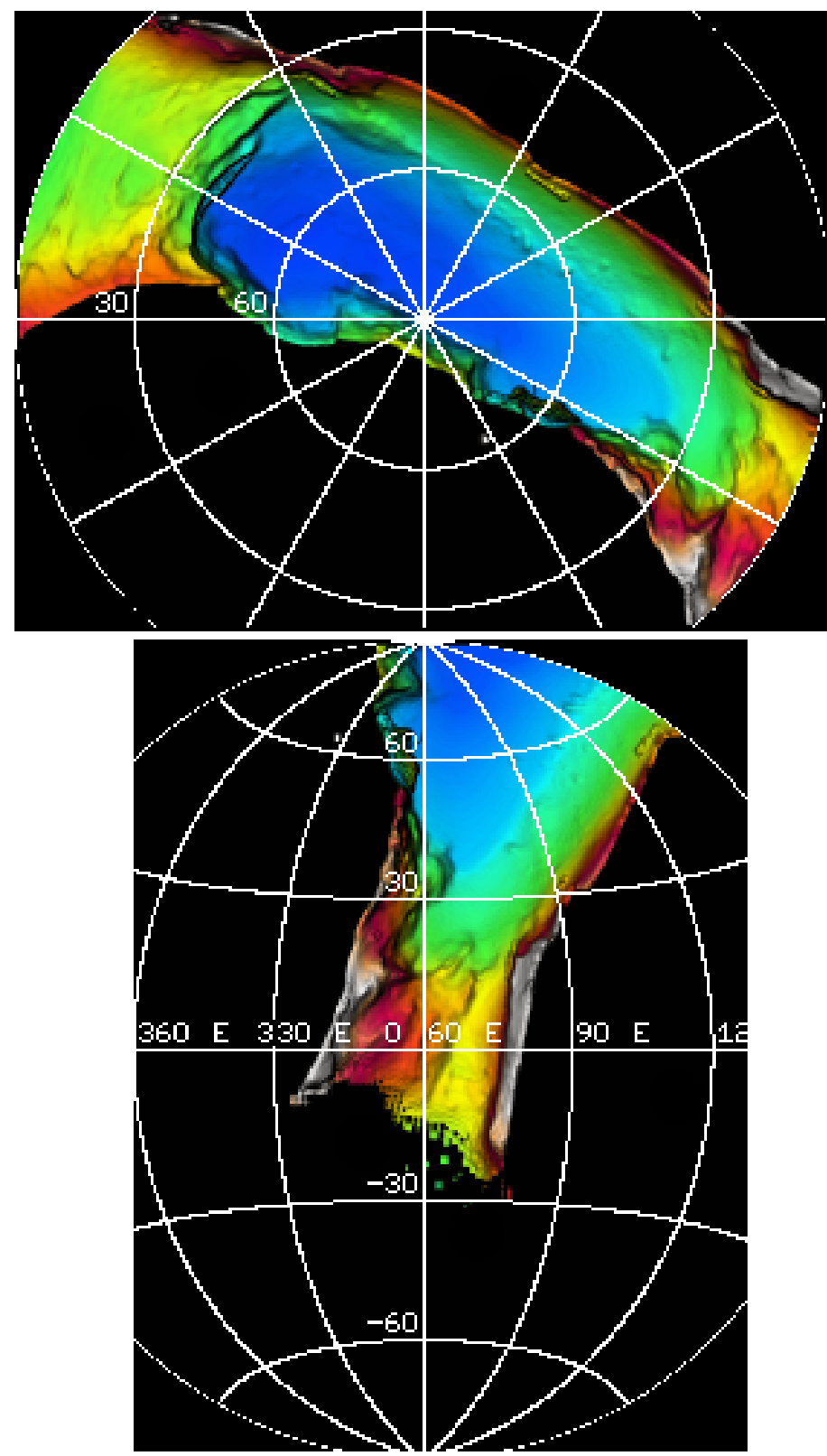
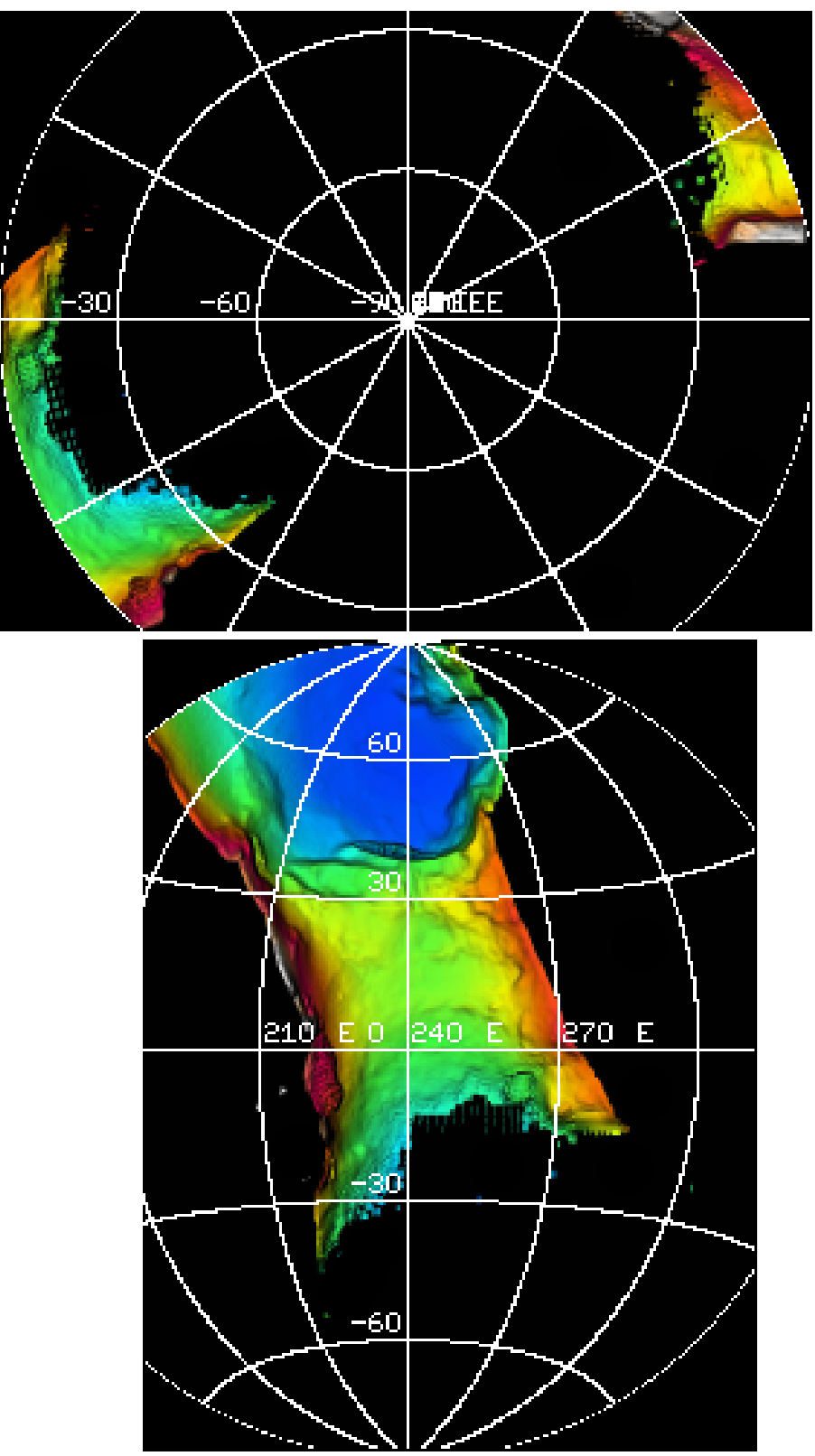

Fig. B.4. Standard maps for the NR (neck region of 67P): if printed in A4 paper format, the map scale is roughly 1:50 K. Height color coding above the $1000 \mathrm{~m}$ reference sphere: blue $=-400 \mathrm{~m}$ to red $/$ white $=400 \mathrm{~m}$. Black areas indicate currently not illuminated unmapped regions on the southern hemisphere of $67 \mathrm{P}$ and cut regions to adjacent sublobes (e.g., at top right and bottom left side in 67P_NR_1000_L_90_0). Top left panel: 67P_NR_1000_L_90_0, top right panel: 67P_NR_1000_L_-90_0, bottom left panel: 67P_NR_1000_L_0_60, bottom right panel: 67P_NR_1000_L_0_240. 